\title{
A New Skeleton Model and the Motion Rhythm Analysis for Human Shoulder Complex Oriented to Rehabilitation Robotics
}

\author{
Song Zhibin (D), Ma Tianyu, Nie Chao, and Niu Yijun \\ Key Laboratory of Mechanism Theory and Equipment Design of Ministry of Education, Tianjin University, Tianjin 300072, China \\ Correspondence should be addressed to Song Zhibin; songzhibin@tju.edu.cn
}

Received 28 November 2017; Revised 23 February 2018; Accepted 13 March 2018; Published 3 June 2018

Academic Editor: Dongming Gan

Copyright ( 2018 Song Zhibin et al. This is an open access article distributed under the Creative Commons Attribution License, which permits unrestricted use, distribution, and reproduction in any medium, provided the original work is properly cited.

\begin{abstract}
Rehabilitation robotics has become a widely accepted method to deal with the training of people with motor dysfunction. In robotics medium training, shoulder repeated exercise training has been proven beneficial for improving motion ability of human limbs. An important and difficult paradigm for motor function rehabilitation training is the movement rhythm on the shoulder, which is not a single joint but complex and ingenious combination of bones, muscles, ligaments, and tendons. The most robots for rehabilitation were designed previously considering simplified biomechanical models only, which led to misalignment between robots and human shoulder. Current biomechanical models were merely developed for rehabilitation robotics design. This paper proposes a new hybrid spatial model based on joint geometry constraints to describe the movement of the shoulder skeletal system and establish the position analysis equation of the model by a homogeneous coordinate transformation matrix and vector method, which can be used to calculate the kinematics of human-robot integrated system. The shoulder rhythm, the most remarkable particularity in shoulder complex kinematics and important reference for shoulder training strategy using robotics, is described and analyzed via the proposed skeleton model by three independent variables in this paper. This method greatly simplifies the complexity of the shoulder movement description and provides an important reference for the training strategy making of upper limb rehabilitation via robotics.
\end{abstract}

\section{Introduction}

Rehabilitation robots have received increasing interest to provide rehabilitative therapy following neurological injuries such as stroke and spinal cord injury [1]. The shoulder is one of the most complex motor function areas of the human body, which has a direct impact on the recovery of upper limb motor function. When a shoulder has motor dysfunction caused by injury or disease, it often needs reasonable rehabilitation suitable for the specific conditions of the patient. For this situation, some studies have shown that a rehabilitation strategy based on task orientation that fuses high intensity repetitive exercise training will be very conducive to the improvement of athletic ability, which is one of the most effective rehabilitation methods [2-7], while the upper limb rehabilitation robot is a universally acknowledged effective way to carry out the rehabilitation strategy.

There are lots of robotics proposed currently for lower limb and upper limb rehabilitation. Most of them got fruitful achievement even though accurate motion information of bones and muscles was not considered thoroughly. There have been many attempts in robotics which evolved from a simplified 3-degrees of freedom (DoF) ball and socket, such as Carignan and Liszka [8] who have defined the shoulder as a 3-DoF ball-and-socket joint. However, it is not effective for a shoulder rehabilitation using exoskeleton robotics which has three orthogonal axes intersecting at one point to assume shoulder as a ball-and-socket joint, because the shoulder cannot be regarded as a single joint but complex and ingenious combination of bones, muscles, ligaments, and tendons. The remarkable characteristic of the shoulder complex is the movement rhythm involving the motion of the scapula, clavicle, and humerus. The rhythm analysis is important and necessary for shoulder complex rehabilitation training.

Biomedical researchers have done a lot of analysis on musculoskeletal models for shoulder training. In 1965, Dempster [9] established a simple two-dimensional series 
link model to describe the relative motion characteristics among bones of the shoulder; however, the model only has existing qualitative description, lacking the description with definition and quantitative analysis of the joint type. Engin and Chen [10] established a three-dimensional 6-DoF rigidbody model of the humerus relative to the human torso in 1986. Furthermore, Engin and Tumer increased the degrees of freedom of the shoulder complex model to 9 in 1989 . Although Engin and Chen established a three-dimensional model of shoulder bones to analyze their kinematic characteristics, the series connection of the shoulder, without exception, simplified the scapula into two connecting rods that are connected to the clavicle and humerus.

There are complicated models in the shoulder joint. Garner and Pandy [11] established a 13-DoF human upper limb skeleton model in 1999. The model does not define the geometric constraints of the scapulothoracic articulation joint (ST) nor analyze the motion of the model. Based on the concept of this model, Maurel and Thalmann and Maurel et al. $[12,13]$ treat the ST joint as a single point contacting with the surface with 5 DoFs, but the study lacks systemic analysis. Tondu [14] proposed a similar shoulder mechanism model in 2005, which defines the ST joint as a planar subordinate on the basis of the Maurel model and ignores the rotational freedom of the sternoclavicular joint (SC) along the direction of the clavicle axis, modeling the shoulder girdle as a 2-DoF spatial parallel mechanism. This model simplifies the difficulty of analyzing shoulder girdles. In order to make the shoulder get a larger workspace and more activity, Lenarcic and Stanisic [15] in 2006 proposed a shoulder girdle skeleton system using a six-legged six-joint parallel mechanism to deal with the simulation, but it is difficult to determine the position of each joint and the length of the parallel bars corresponding to the actual shoulder girdle. It is also difficult to correspond to the movement of a single individual bone.

The current rehabilitation robots were designed with a simplified skeleton model and set the degrees of freedom such as the robotic-arm exoskeleton proposed by Klein et al. [16]. Therefore, this paper provides a suitable model that describes the shoulder complex motion via a model of a spatial hybrid mechanism with four rods and four joints and verifies the accuracy of the model through real data for rehabilitation robots in the shoulder joint. Among them, SC joint, acromioclavicular (AC) joint, and glenohumeral (GH) joint are all defined as spherical hinge joints, and the thorax is approximated as an ellipsoid. The constraint of the ST joint is defined as the two fixed points on the suprascapular connected to the thorax ellipsoid in a point contact constraint, and the rotation of the SC joint around the clavicular axis is considered to be an extra DoF.

\section{Modeling and Analysis}

In the model of the shoulder musculoskeletal dynamics, the posture analysis of the bones constituting the shoulder is the basis for shoulder motion analysis, muscle drive characteristics, muscle strength, and joint force analysis. Due to the complexity of the shoulder skeletal system, it is difficult

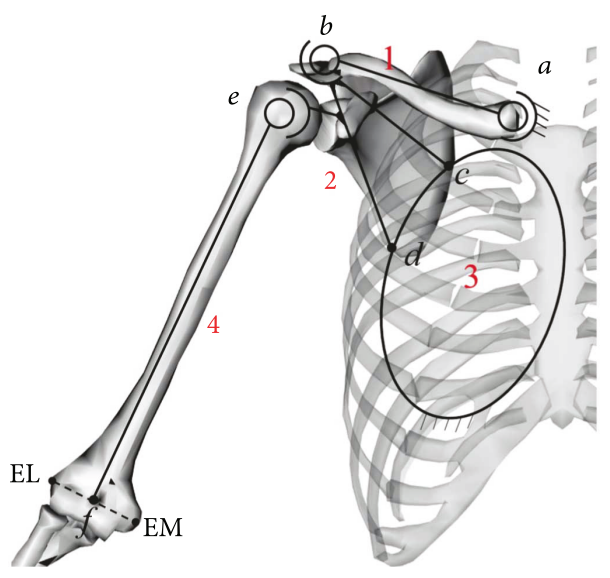

Figure 1: Schematic diagram of the shoulder mechanism.

to obtain the people's posture of the shoulder bone quickly by using the commonly used real-time skeleton marking method. Based on results provided by Garner and Pandy $[11,17]$ for the anatomical results of human shoulder geometry, the human shoulder skeleton system is simplified as a hybrid spatial mechanism model to simulate and analyze the movement of the shoulder bone. The motions of each joint and bone are described by defining the local coordinate systems fixed on the corresponding bones, and the position inverse analysis of the mechanism is completed by the homogeneous coordinate transfer matrix and vector method, which simplified the analysis and prediction of the shoulder skeleton configuration. Finally, this chapter verifies the rationality of the model through the experiment of the actual human shoulder.

2.1. A Proposed Model of Shoulder Skeleton System. The kinematics of the shoulder of the human body mainly depends on the skeleton system composed of the shoulder girdle and humerus compared with the muscle system and the tendon system, so in this paper, the skeleton system for the shoulder is mainly discussed. It can be considered a closed-loop rigid system composed of the thorax, clavicle, and scapula. Joints involved in shoulder movement involve the AC joint, the SC joint, the ST joint, and the GH joint.

In this paper, a hybrid spatial mechanism model is used to simulate the shoulder skeletal system, as shown in Figure 1. In this model, the SC joint, the AC joint, and the $\mathrm{GH}$ joint are denoted as $a, b$, and $e$, respectively, and are treated as the ideal 3-DoF ball-and-socket joint. The scapula achieves two-point contact with the thoracic ellipsoid surface through its medial lateral edge $c$ and $d$, which may be equivalent to cylindrical-planar pairs with 4 DoFs. Among them, components 1,2,3, and 4 represent the clavicle, the scapula, the thoracic surface, and the humerus of the upper arm, respectively. The long axis of the humerus represents component 4 , that is, the direction of connecting the center of the GH joint $e$ and the center of the elbow $f$, where the elbow joint $f$ is defined as the midpoint of the outer lateral ankle EL and the inside ankle EM. 
2.2. Kinematics Analysis of the Proposed Model. In the above mechanism model, components 1, 2, 3, and 4 constitute a hybrid spatial mechanism containing a closed chain and an open chain, where components 1,2 , and 3 form a closedloop chain.

2.2.1. To Calculate DoFs of the Proposed Mechanism. To obtain the kinematics of the mechanism, we should first analyze its DoFs. For the closed loop of the shoulder girdle, the DoF of the mechanism $(M)$ is obtained by the Kutzbach-Grubler formula [18].

$$
M=6(n-g-1)+\sum_{i=1}^{g} f_{i},
$$

where $n$ is the number of mechanism components, $g$ is the number of joints, and $f_{i}$ is the relative freedom of the $i t h$ motion pair. The closed-loop part has three components and three motion pairs. Both SC and AC are ball-andsocket joints with $3 \mathrm{DoF}$. The ST joint is a cylinder-plane pair with 4 DoF. So a closed chain with $4 \mathrm{DoF}$ in the shoulder joint system can be obtained. Considering the humerus movement, the total degree of freedom of the shoulder is up to 7 . However, in the process of activity of the shoulder, the rotation of the clavicle around its axis is very small and it has an internal DoF that does not change the whole posture of link 4 [19-21]. Therefore, for joint $a$, the rotation in the direction of the winding of component 2 is an extra freedom. If the rotation angle is known, the motion posture of the shoulder girdle can be obtained by inputting three joint variables.

2.2.2. To Establish Shoulder Global Coordinate and Local Coordinates Fixed to a Single Bone. In order to describe and analyze the kinematics of the skeleton model of the shoulder complex, every bone is fixed to one coordinate system, as shown in Figure 2. The origin point of the global coordinate system $S_{o}=\left\{a-x_{0} y_{0} z_{0}\right\}$ is the SC joint at point $a$, and axes $x_{0}, y_{0}$, and $z_{0}$ are, respectively, parallel to three intersections of the human anatomical coronal plane, the sagittal plane, and the transverse plane. For the clavicle system $S_{1}=$ $\left\{a-x_{1} y_{1} z_{1}\right\}$, the origin is located on the SC joint at point $a$. The $z_{1}$ axis is in the direction of the clavicle axis, and the $x_{1}$ axis is defined on the horizontal plane and perpendicular to $z_{1}$. For the scapular system $S_{2}=\left\{b-x_{2} y_{2} z_{2}\right\}$, its origin point is located at the point of the AC joint at $b$, where the $z_{2}$ axis is in the direction from $b$ to the medial margin of the scapula $c$ and the direction of $x_{2}$ is perpendicular to the plane determined by points $b, c$, and $d . S_{3}$ is parallel to $S_{0}$. For the humeral coordinate system $S_{4}=\left\{e-x_{4} y_{4} z_{4}\right\}$, the origin point is located at the center $e$ of the glenoid joint, where the $z_{4}$ axis is in the direction from $f$ to $e$; the $y_{4}$ axis is perpendicular to the plane determined by point $e$, EL, and EM; and the $x_{4}$ axis is determined by the right-hand rule.

2.2.3. Kinematics Analysis of the Joints in the Shoulder. In the shoulder girdle, the thorax, the clavicle, and the scapula are interconnected with each other through the joints SC, AC, and ST, thus forming a closed-loop mechanism. For the

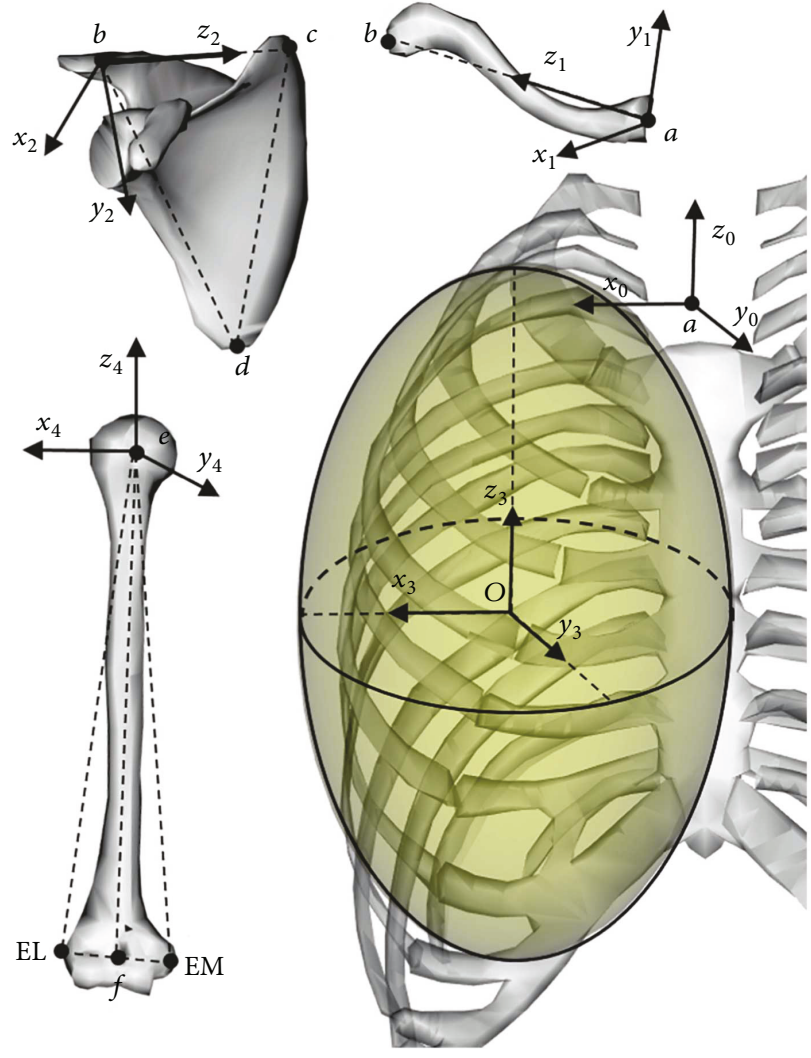

FIGURE 2: Shoulder bony segments and related reference frames.

closed-chain mechanism, position analysis can be attributed to determine the remaining joint variables and skeletal posture using the known mechanism geometric parameters and at least three joint variables as inputs. In this paper, the purpose of inverse analysis is to verify the accuracy of the actual shoulder movement predicted by the model.

In order to describe the matrix conveniently, the distance between joint $a$ and joint $b$ is set as $l_{1}$, the distance between the center of joint $b$ and point $c$ is $l_{2}$, the distance between $b$ and point $d$ is $l_{3}$, and the length of the humerus is $l_{4}$.

According to the above definition for the coordinate system, transition from the system $S_{o}=\left\{a-x_{0} y_{0} z_{0}\right\}$ to $S_{1}=\left\{a-x_{1} y_{1} z_{1}\right\}$, namely, the motion of the thorax relative to the thorax, can be expressed via the following matrix:

$$
\begin{aligned}
{ }_{1}^{0} \mathbf{T} & =\operatorname{Rot}\left(z, \theta_{1}\right) \operatorname{Rot}\left(y, \theta_{2}\right) \operatorname{Rot}\left(z, \theta_{3}\right) \\
& =\left[\begin{array}{cccc}
c \theta_{1} c \theta_{3}-s \theta_{1} c \theta_{2} s \theta_{3} & -c \theta_{1} s \theta_{3}-s \theta_{1} c \theta_{2} c \theta_{3} & s \theta_{1} s \theta_{2} & 0 \\
s \theta_{1} c \theta_{3}+c \theta_{1} c \theta_{2} s \theta_{3} & -s \theta_{1} s \theta_{3}+c \theta_{1} c \theta_{2} c \theta_{3} & -c \theta_{1} s \theta_{2} & 0 \\
s \theta_{2} s \theta_{3} & s \theta_{2} c \theta_{3} & c \theta_{2} & 0 \\
0 & 0 & 0 & 1
\end{array}\right],
\end{aligned}
$$

where s and c denote sine and cosine, respectively.

The conversion from system $S_{1}$ to system $S_{2}$ can be assumed to have moved $l_{1}$ along the $z$-axis firstly and rotated 
$\theta_{4}$ around the $y$-axis, then rotated $\theta_{5}$ around the $x$-axis, and finally rotated $\theta_{5}$ around the $y$-axis. The conversion matrix is

$$
\begin{aligned}
{ }_{2}^{1} \mathbf{T} & =\operatorname{Trans}\left(z, l_{1}\right) \operatorname{Rot}\left(y, \theta_{4}\right) \operatorname{Rot}\left(x, \theta_{5}\right) \operatorname{Rot}\left(y, \theta_{6}\right) \\
& =\left[\begin{array}{cccc}
\mathrm{c} \theta_{4} \mathrm{c} \theta_{6}-\mathrm{s} \theta_{4} \mathrm{c} \theta_{5} \mathrm{~s} \theta_{6} & \mathrm{~s} \theta_{4} \mathrm{~s} \theta_{5} & \mathrm{c} \theta_{4} \mathrm{~s} \theta_{6}+\mathrm{s} \theta_{4} \mathrm{c} \theta_{5} \mathrm{c} \theta_{6} & 0 \\
\mathrm{~s} \theta_{5} \mathrm{~s} \theta_{6} & \mathrm{c} \theta_{5} & -\mathrm{s} \theta_{5} \mathrm{c} \theta_{6} & 0 \\
-\mathrm{s} \theta_{4} \mathrm{c} \theta_{6}-\mathrm{c} \theta_{4} \mathrm{c} \theta_{5} \mathrm{~s} \theta_{6} & \mathrm{c} \theta_{4} \mathrm{~s} \theta_{5} & -\mathrm{s} \theta_{4} \mathrm{~s} \theta_{6}+\mathrm{c} \theta_{4} \mathrm{c} \theta_{5} \mathrm{c} \theta_{6} & l_{1} \\
0 & 0 & 0 & 1
\end{array}\right] .
\end{aligned}
$$

The position and scale of the thoracic ellipsoid are known. Assuming that the position of the ellipsoid center point $O$ in the fixed system $S_{0}$ is ${ }^{0} P_{O}=\left[x_{0}, y_{0}, z_{0}, 1\right]^{T}$, the transfer matrix of ellipsoid $S_{3}$ relative to fixed system $S_{0}$ is

$$
{ }_{3}^{0} \mathbf{T}=\left[\begin{array}{llll}
1 & 0 & 0 & x_{0} \\
0 & 1 & 0 & y_{0} \\
0 & 0 & 1 & z_{0} \\
0 & 0 & 0 & 1
\end{array}\right]
$$

If the semilong axes of the ellipsoid are, respectively, $m, n$, and $p$ and point $c$ and point $d$ are located in the thoracic ellipsoid table surface, the position coordinates can be represented by the parametric equation of the ellipsoid:

$$
\begin{aligned}
& { }^{3} P_{c}=\left[\begin{array}{llll}
m \sin \varphi_{c} \cos \varphi_{c} & n \sin \varphi_{c} \cos \varphi_{c} & p \cos \varphi_{c} & 1
\end{array}\right]^{T}, \\
& { }^{3} P_{d}=\left[\begin{array}{llll}
m \sin \varphi_{d} \cos \varphi_{d} & n \sin \varphi_{d} \cos \varphi_{d} & p \cos \varphi_{d} & 1
\end{array}\right]^{T},
\end{aligned}
$$

where $\varphi_{c}, \phi_{c}, \varphi_{d}$, and $\phi_{d}$ in the formula, respectively, describes the position parameters of point $c$ and point $d$. Point $c$ and point $d$ are also located on the shoulder blade, as shown in Figure 3. The position coordinates of $c$ and $d$ in the scapula system are

$$
\begin{aligned}
{ }^{2} P_{c} & =\left[\begin{array}{llll}
0 & 0 & l_{2} & 1
\end{array}\right]^{T}, \\
{ }^{2} P_{d} & =\left[\begin{array}{llll}
0 & l_{3} \sin \gamma & l_{3} \cos \gamma & 1
\end{array}\right]^{T},
\end{aligned}
$$

where $\gamma$ in the formula is the included angle of $\overrightarrow{b c}$ and $\overrightarrow{b d}$, because points $b, c$, and $d$ are all on the scapula, and $l_{2}, l_{3}$, and $\gamma$ are constant. In summary, using the location transform relationship of $c$ and $d$, the establishment of displacement constraint equation is as follows:

$$
\begin{aligned}
& { }^{0} P_{c}={ }_{1}^{0} T_{2}^{1} T^{2} p_{c}={ }_{3}^{0} T^{3} p_{c}, \\
& { }^{0} P_{d}={ }_{1}^{0} T_{2}^{1} T^{2} p_{d}={ }_{3}^{0} T^{3} p_{d} .
\end{aligned}
$$

Formula (8) above can be expressed as follows:

$$
\begin{aligned}
& { }_{1}^{0} T_{2}^{1} T^{2} p_{c}-{ }_{3}^{0} T^{3} p_{c}=0, \\
& { }_{1}^{0} T_{2}^{1} T^{2} p_{d}-{ }_{3}^{0} T^{3} p_{d}=0 .
\end{aligned}
$$

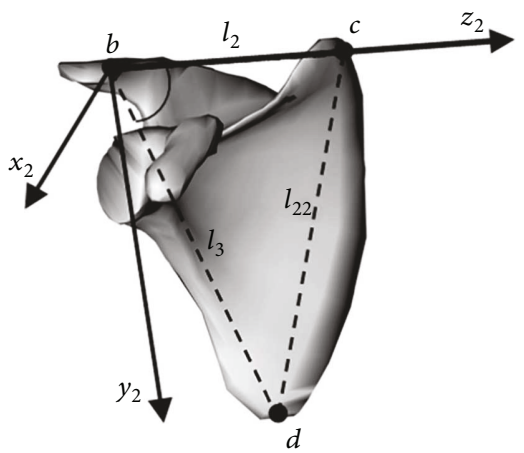

Figure 3: Points $c$ and $d$ located on the scapula.

Equation (9) is the joint position solution constraint equation of the girdle mechanism. Let each component be zero; the equations can be transformed into 6 constraint equations which contain a total of $\theta_{1} \sim \theta_{6}, \varphi_{c}, \phi_{c}, \varphi_{d}, \phi_{d} 10$ joint variables. Clavicle rotation around its own axis $z_{1}$ is an extra degree of freedom, and if $\theta_{3}$ is zero, just any three rotational variables can be used as inputs to solve the remaining variables.

The kinematic analysis of the shoulder girdle is completed, while the humerus is connected to the shoulder complex through the $\mathrm{GH}$ joint as the end of execution. The motion posture of the upper arm is usually studied in the global reference system, using the ISB standard recommended by $\mathrm{Wu}$ and Cavanagh $[22,23]$ to describe the posture of the humerus. The rotation matrix applied to convert the global system $S_{0}$ into the humerus system $S_{4}$ is

$$
\begin{aligned}
{ }_{4}^{0} \mathbf{R} & =\operatorname{Rot}\left(y, \theta_{7}\right) \operatorname{Rot}\left(x, \theta_{8}\right) \operatorname{Rot}\left(z, \theta_{9}\right) \\
& =\left[\begin{array}{cccc}
c \theta_{7} c \theta_{9}-s \theta_{7} c \theta_{8} s \theta_{9} & s \theta_{7} s \theta_{8} & c \theta_{7} c \theta_{9}+s \theta_{7} c \theta_{8} s \theta_{9} & 0 \\
s \theta_{8} s \theta_{9} & c \theta_{8} & -s \theta_{8} c \theta_{9} & 0 \\
-s \theta_{7} c \theta_{9}-c \theta_{7} c \theta_{8} s \theta_{6} & c \theta_{7} s \theta_{8} & -s \theta_{7} c \theta_{9}+c \theta_{7} c \theta_{8} s \theta_{9} & 0 \\
0 & 0 & 0 & 1
\end{array}\right] .
\end{aligned}
$$

If the position of the $\mathrm{GH}$ center coordinates ${ }^{0} P_{e}=$ $\left[\begin{array}{llll}x_{e} & y_{e} & z_{e} & 1\end{array}\right]^{T}$ is given, the conversion matrix for the translation from the humeral system $S_{0}$ to the global system $S_{4}$ is

$$
\left[\begin{array}{cccc}
\mathrm{c} \theta_{7} \mathrm{c} \theta_{9}-\mathrm{s} \theta_{7} \mathrm{c} \theta_{8} \mathrm{~s} \theta_{9} & \mathrm{~s} \theta_{7} \mathrm{~s} \theta_{8} & \mathrm{c} \theta_{7} \mathrm{c} \theta_{9}+\mathrm{s} \theta_{7} \mathrm{c} \theta_{8} \mathrm{~s} \theta_{9} & x_{e} \\
\mathrm{~s} \theta_{8} \mathrm{~s} \theta_{9} & \mathrm{c} \theta_{8} & -\mathrm{s} \theta_{8} \mathrm{c} \theta_{9} & y_{e} \\
-\mathrm{s} \theta_{7} \mathrm{c} \theta_{9}-\mathrm{c} \theta_{7} \mathrm{c} \theta_{8} \mathrm{~s} \theta_{6} & \mathrm{c} \theta_{7} \mathrm{~s} \theta_{8} & -\mathrm{s} \theta_{7} \mathrm{c} \theta_{9}+\mathrm{c} \theta_{7} \mathrm{c} \theta_{8} \mathrm{~s} \theta_{9} & z_{e} \\
0 & 0 & 0 & 1
\end{array}\right] .
$$

2.3. Verification for Shoulder Skeletal Motion Based on Reference Data. In this paper, the size of shoulder bones 
TABLE 1: The size parameters of each link of mechanism model.

\begin{tabular}{lccccc}
\hline$l_{1}$ & $l_{2}$ & $l_{3}$ & $l_{22}$ & $l_{4}$ & $\gamma$ \\
\hline $154.17 \mathrm{~mm}$ & $120.71 \mathrm{~mm}$ & $187.21 \mathrm{~mm}$ & $109.83 \mathrm{~mm}$ & $301.56 \mathrm{~mm}$ & $33.8^{\circ}$ \\
\hline
\end{tabular}

refers to the anatomical data of human upper arm bones and joints obtained by Garner and Pandy [11, 17, 24]. Shoulder Database V1.1, published by Bolsterlee et al. [21] in 2014, is used as a reference source of shoulder motion data. Then, through the joint displacement constraint equations obtained above, the movement of the mechanism is analyzed by using Matlab.

2.3.1. Shoulder Mechanism Geometry. In this paper, the size of the mechanism is based on the anatomy of human bones and joints obtained by Garner and Pandy; the size of each component shown in Figure 1 is as shown in Table 1 below. The semimajor axis of the thorax ellipsoid and the center point of its ellipsoid are shown in Table 2. The coordinates of the center point are in the fixed system $S_{0}$.

The position of GH joint center in the scapular $S_{2}$ is ${ }^{2} p_{e}=\left[\begin{array}{llll}-0.29 & 43.71 & 3.41 & 1\end{array}\right]^{T}$.

2.3.2. Description of Shoulder Joint Movement. The local coordinate system of each skeleton in Figure 2 is defined to be calculated conveniently. The ISB standards recommended by $\mathrm{Wu}$ and Cavanagh and $\mathrm{Wu}$ et al. $[22,23]$ are widely used in biomedical engineering to describe the human upper body bones and joints in the movement. In the ISB standard, the local coordinate system of the shoulder bones and the bony landmark are shown in Figure 4.

In this paper, the shoulder skeletal configuration analysis results and experimental measurements which are unified according to the ISB standard will be compared; therefore, it is necessary to figure out the corresponding relationship between the calculation of the coordinate system and the ISB coordinate system. Klein Breteler et al. [25] obtained a more favorable shoulder morphological datasets by dissecting the body of a 57-year-old male and establishing a universal dataset of human shoulder bones. Table 3 shows the relevant parameters of bone markers and the size of the thorax of the general model and the experimental subjects in this article. All the coordinates are converted corresponding to the thorax system; the length unit is $\mathrm{mm}$.

Through the definition of each local coordinate system and the characteristic sizes of each marked point of the shoulder skeleton in Tables 2 and 3, the relationship between the coordinate systems $S_{0}, S_{1}, S_{2}$, and $S_{4}$ used in the above calculation and each coordinate system $S_{t}, S_{c}, S_{s}$, and $S_{h}$ recommended in ISB standard can be described by the following rotation transformation matrix:

$$
{ }_{0}^{t} \mathbf{R}=\left[\begin{array}{ccc}
0 & 1 & 0 \\
0 & 0 & 1 \\
1 & 0 & 0
\end{array}\right]
$$

TABLE 2: The size parameters of thorax ellipsoid ( $\mathrm{mm})$.

\begin{tabular}{lcccccc}
\hline$m$ & $n$ & $p$ & \multicolumn{4}{c}{${ }^{0} p_{\mathrm{o}}$} \\
\hline 76.85 & 97.17 & 217.44 & {$\left[\begin{array}{lllll}28.12 & -43.90 & -164.51 & 1\end{array}\right]^{T}$} \\
\hline
\end{tabular}

$$
\begin{aligned}
{ }_{1}^{c} \mathbf{R} & =\left[\begin{array}{lll}
1 & 0 & 0 \\
0 & 1 & 0 \\
0 & 0 & 1
\end{array}\right], \\
{ }_{2}^{s} \mathbf{R} & =\left[\begin{array}{lll}
0.9599 & 0.0373 & -0.2778 \\
-0.0131 & -0.9841 & -0.1773 \\
-0.2800 & -0.1738 & -0.9441
\end{array}\right], \\
{ }_{4}^{h} \mathbf{R} & =\left[\begin{array}{lll}
0 & 1 & 0 \\
0 & 0 & 1 \\
1 & 0 & 0
\end{array}\right] .
\end{aligned}
$$

2.3.3. Definition of Shoulder Experimental Motion. Shoulder Database (Shoulder Database V1.1) published by Bolsterlee et al. in 2014 provides data of shoulder motion which has been used in the analysis $[21,26]$. The experiment collected data from five healthy adult males. This article selects one of the objects $S_{1}$ (age 29, height $186 \mathrm{~cm}$, and weight $109 \mathrm{~kg}$ ) from the database. The experiment used a set of motion detection system (Optotrak System, Northern Digital Inc., Waterloo, Ontario, Canada) to detect the spatial positions of six marker groups fixed to the thorax, scapula, humerus, forearm, and palm shown in Figure 5. The locations of the bone markers are arranged according to ISB standard, and the series of the position of the markers are collected at a frequency of $100 \mathrm{~Hz}$.

In order to get accurate shoulder movement data, during the experiment, the subject was asked to complete the range of motion (ROM) tasks including shoulder abduction (ABD), flexion (FLEX), and scapular plane movement (SCAP) which contain all the basic movement of the shoulder shown in Table 4 . With processing, the data of the movement posture of each bone in the shoulder can be uniformly converted into the value in $S_{t}$.

2.3.4. The Solution and Verification for Posture and Position of Each Bone. Through the processing of the spatial coordinates of each marker measured in the experiment, the joint variables $\theta_{1}, \theta_{2}, \phi_{d}$ are obtained, and the three independent joint displacements are used as input, and then the remaining six joint variables $\theta_{4}, \theta_{5}, \theta_{6}, \varphi_{d}, \phi_{c}, \varphi_{c}$ are obtained, as well as the shoulder girdle movement gesture, which is finally compared with the experimental measurement results. 


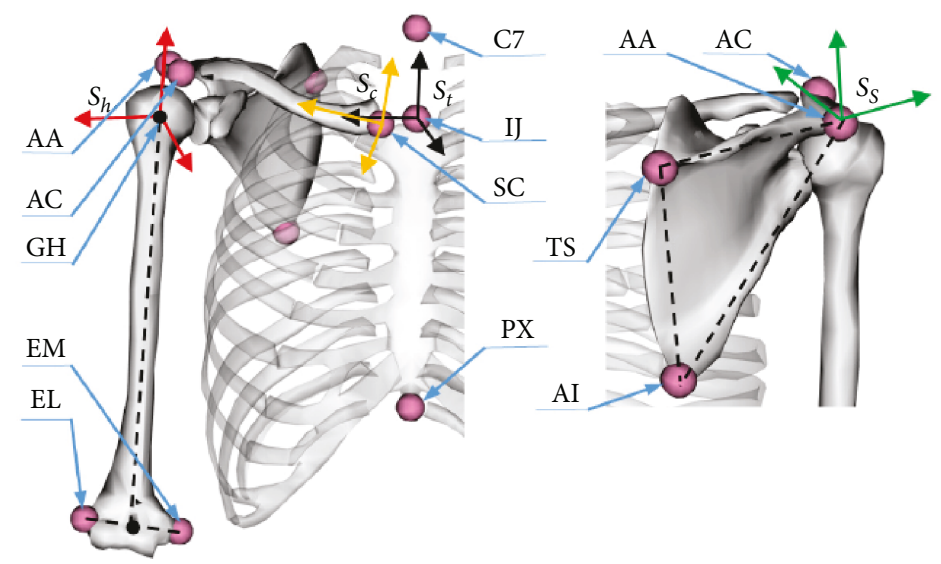

FIGURE 4: The bony landmarks and local coordinate systems defined in ISB.

TABLE 3: The skeletal feature size parameters of the experimental subjects $(\mathrm{mm})$.

\begin{tabular}{lcc}
\hline Bone markers & Common parameters & Test subject \\
\hline IJ & $(0,0,0)$ & $(0,0,0)$ \\
PX & $(31.9,-132.7,-9.8)$ & $(56.0,-163.0,-2.4)$ \\
C7 & $(-124.2,54.1,0.0)$ & $(-123.1,80.7,-4.7)$ \\
T8 & $(-156.61,-171.5,0.0)$ & $(-179.1,-133.2,11.2)$ \\
AA & $(-105.6,7.5,182.6)$ & $(-73.5,9.3,205.1)$ \\
TS & $(-156.0,-11.7,75.0)$ & $(-136.3,5.1,121.8)$ \\
AI & $(-156.7,-126.2,101.9)$ & $(-141.6,-126.5,116.2)$ \\
AC & $(-71.8,26.6,165.1)$ & $(-37.0,40.4,167.2)$ \\
SC & $(-2.8,-15.2,1.4)$ & $(15.3,-31.0,-6.6)$ \\
Ellipsoid center & $(-62.1,-152.1,0.0)$ & $(-61.6,-148.1,0.0)$ \\
Ellipsoidal & $(95.6,211.7,144.6)$ & $(114.4,222.7,164.8)$ \\
semimajor axis & & $(1.20,1.05,1.14)$ \\
Zoom factor & &
\end{tabular}

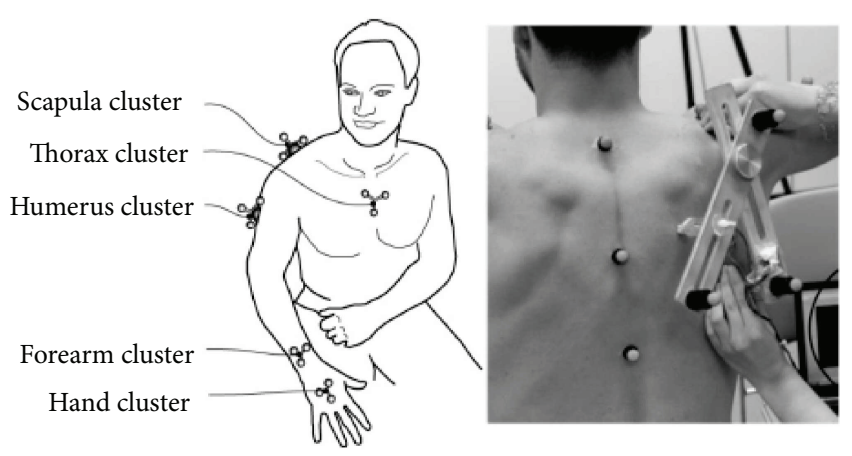

Figure 5: Placement of clusters of Optotrak markers.

Figure 6 shows the thorax movement gesture of the experimental subject $S_{1}$ in the ABD exercise test, and the posture of the humerus under YXY rotation sequence is described by three corresponding Euler angles HumY ${ }_{1}$, HumX, and HumY $\mathrm{H}_{2}$. Figure 7 shows the measured posture of the clavicle relative to the thorax, and according to ISB standard, the posture of the clavicle is described under
YXZ rotation sequence. The three Euler angles formed that depended on them are ClavY, ClavX, and ClavZ, respectively.

Thus, at any time, the posture matrix of the clavicle relative to the thorax can be obtained:

$$
{ }_{c}^{t} \mathbf{R}=\operatorname{Rot}(y, \operatorname{ClavY}) \operatorname{Rot}(x, \operatorname{ClavX}) \operatorname{Rot}(z, \operatorname{ClavZ}) .
$$

Suppose that

$$
{ }_{c}^{t} \mathbf{R}=\left[\begin{array}{lll}
u_{t c x} & v_{t c x} & w_{t c x} \\
u_{t c y} & v_{t c y} & w_{t c y} \\
u_{t c z} & v_{t c z} & w_{t c z}
\end{array}\right] .
$$

Substitute (17) into (12) and (13):

$$
{ }_{1}^{0} R=\left({ }_{0}^{t} R\right){ }^{-1}{ }_{c}^{t} R_{1}^{c} R=\left[\begin{array}{ccc}
u_{z} & v_{z} & w_{z} \\
u_{x} & v_{x} & w_{x} \\
u_{y} & v_{y} & w_{y}
\end{array}\right] .
$$

Let the third column of (18) and (2) be the same, and the following three constraint equations can be obtained:

$$
\begin{aligned}
\mathrm{s} \theta_{1} \mathrm{~s} \theta_{2} & =w_{z}, \\
-\mathrm{c} \theta_{1} \mathrm{~s} \theta_{2} & =w_{x}, \\
\mathrm{c} \theta_{2} & =w_{y}
\end{aligned}
$$

then

$$
\begin{aligned}
& \theta_{1}=\tan ^{-1}\left(-\frac{w_{z}}{w_{x}}\right), \\
& \theta_{2}=\tan ^{-1}\left(\frac{w_{z}}{w_{y} \mathrm{~s} \theta_{1}}\right) .
\end{aligned}
$$

Figure 8 is the result of the input joint variables $\theta_{1}$ and $\theta_{2}$.

Assuming that the object $S_{1}$ is at a certain moment of ABD movement, the position vector of the sternoclavicular joint marking point SC is ${ }^{t} \mathbf{p}_{\mathrm{sc}}$, and the conversion 
TABLE 4: Range of motion (ROM) tasks.

Shoulder movement

ABD (shoulder abduction)

FLEX (shoulder anteflexion)

SCAP (shoulder scaption)
Motion description

Motion in the frontal plane. Initially, the arm relaxes and hangs down and then moves in the frontal plane, until the humerus rises up to the extreme position, and then moves back to the initial location. Keep the palm facing the body and upper limb unbent during exercises.

Flexion movement in the sagittal plane. Initially, the arm relaxes and hangs down and then flexes in the sagittal plane, until the humerus rises up to the extreme position, and then moves back to the initial location. Movement in the scapular plane. Initially, the arm relaxes and hangs down and then raises the scapular plane (at about $45^{\circ}$ to the frontal plane) until the humerus rises up to the extreme position and then back to the initial position.

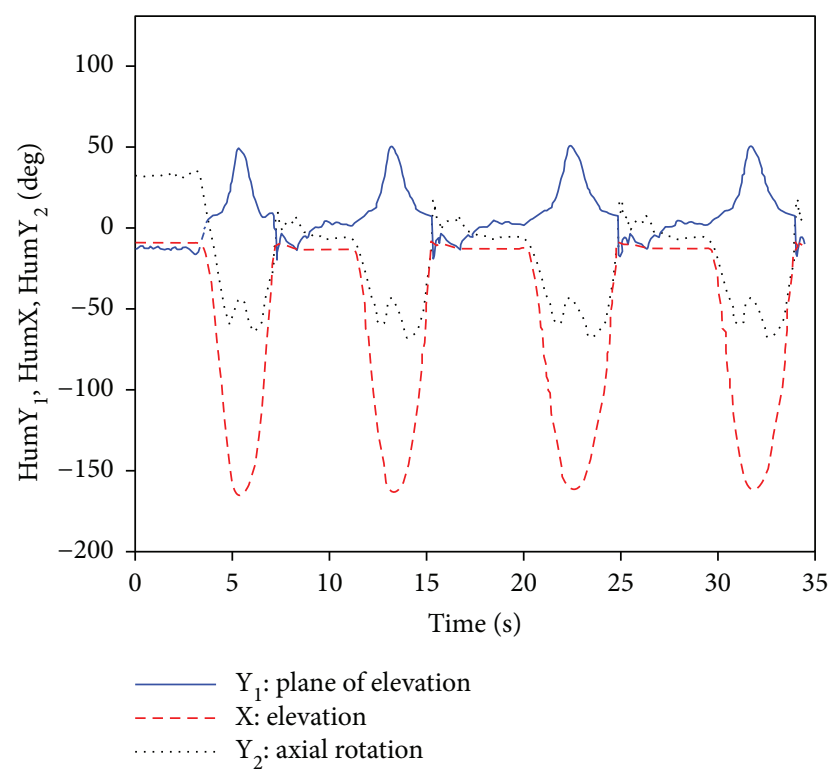

Figure 6: Movement posture of the humerus (ABD).

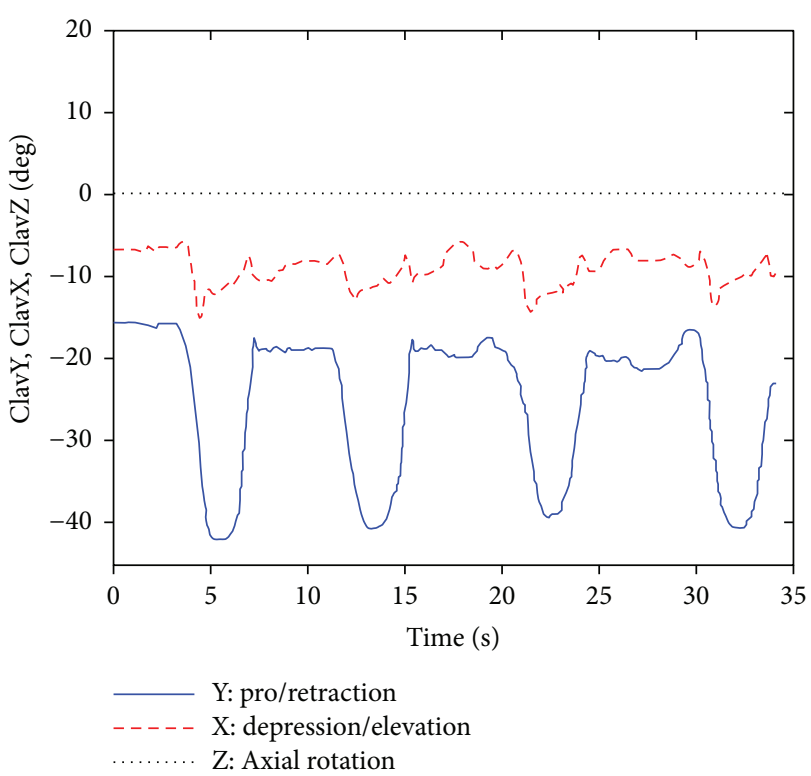

Figure 7: The posture of the clavicle that is adopted to be input parameters (ABD).

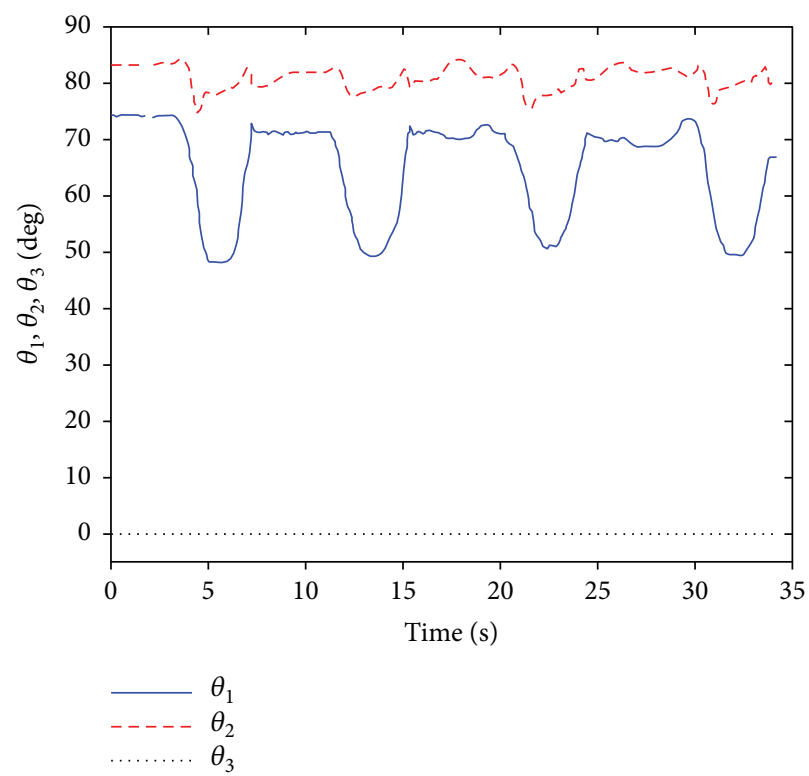

FIgURE 8: The joint angles $\theta_{1}$ and $\theta_{2}$ that are adopted to be input parameters (ABD).

relationship between $S_{t}$ and $S_{0}$ can be expressed as the following transfer matrix:

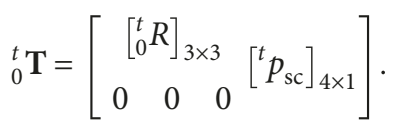

Thus, the position vector of the lower scapular AI in the ellipsoidal system $S_{3}$ can be obtained:

$$
{ }^{3} \mathbf{p}_{\mathrm{AI}}=\left({ }_{3}^{t} T\right)^{-1}{ }^{t} p_{A I}=\left[\begin{array}{llll}
x_{A I, 3} & y_{A I, 3} & z_{A I, 3} & 1
\end{array}\right]^{T} .
$$

Combining formulas (4) and (12) and Table 3 on the thorax data, we can have the following formula:

$$
{ }_{3}^{t} T={ }_{0}^{t} T_{3}^{0} T=\left[\begin{array}{cccc}
0 & 1 & 0 & -61.6 \\
0 & 0 & 1 & -148.1 \\
1 & 0 & 0 & 0 \\
0 & 0 & 0 & 1
\end{array}\right] .
$$

In the ABD exercise of the experimental subject $S_{1}$, the position of the lower scapular angle AI in the ellipsoid $S_{3}$ is shown in Figure 9. 


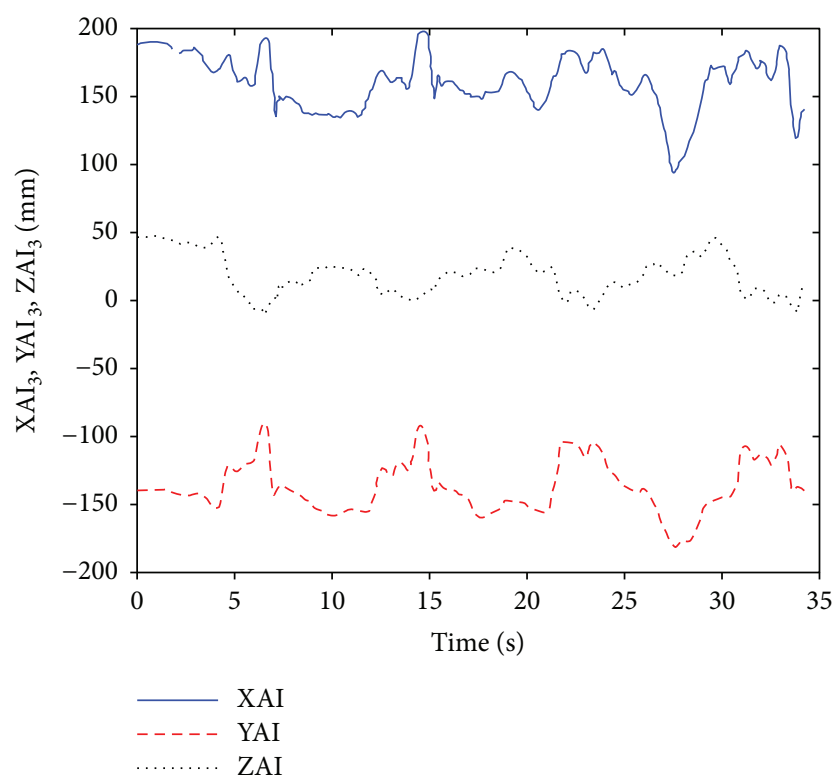

FIgURE 9: The position vector of AI described in ellipsoid frame $S_{3}$.

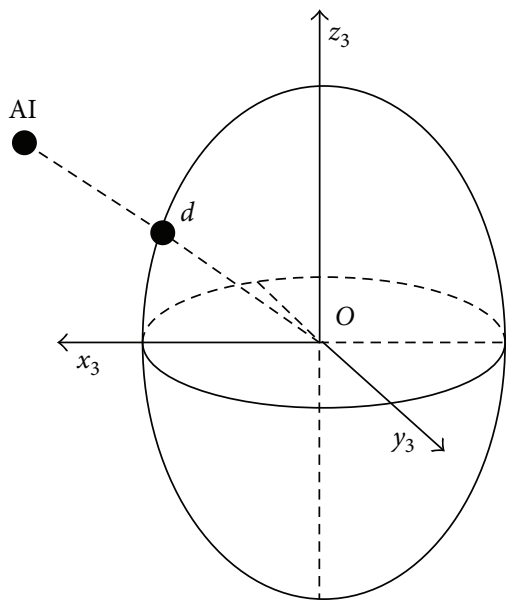

FIGURE 10: Point AI projects on the elliptical surface.

However, the fact is that the AI point is located on the shoulder blade but not always on the surface of the ellipsoid, which means it does not exactly coincide with point $d$ in the model. In this paper, the projection point of the subscapular AI along the centerline of the ellipsoid is regarded as the equivalent point $d$. As shown in Figure 10, the connection between AI and ellipsoid center $O$ intersects at $d$ on the ellipsoid surface, and the position and size of the chest ellipsoid of the experimental subject $S_{1}$ refer to Table 3 .

It can be obtained that $\overrightarrow{O d} \| \overrightarrow{O A I}$. Substitute (6) and (22), then there is

$$
\frac{b \sin \varphi_{d} \sin \phi_{d}}{a \sin \varphi_{d} \cos \phi_{d}}=\frac{y_{\mathrm{AI}, 3}}{x_{\mathrm{AI}, 3}} .
$$

Another input joint variable can be found:

$$
\phi_{d}=\tan ^{-1}\left(\frac{a}{b} \frac{y_{\mathrm{AI}, 3}}{x_{\mathrm{AI}, 3}}\right) .
$$

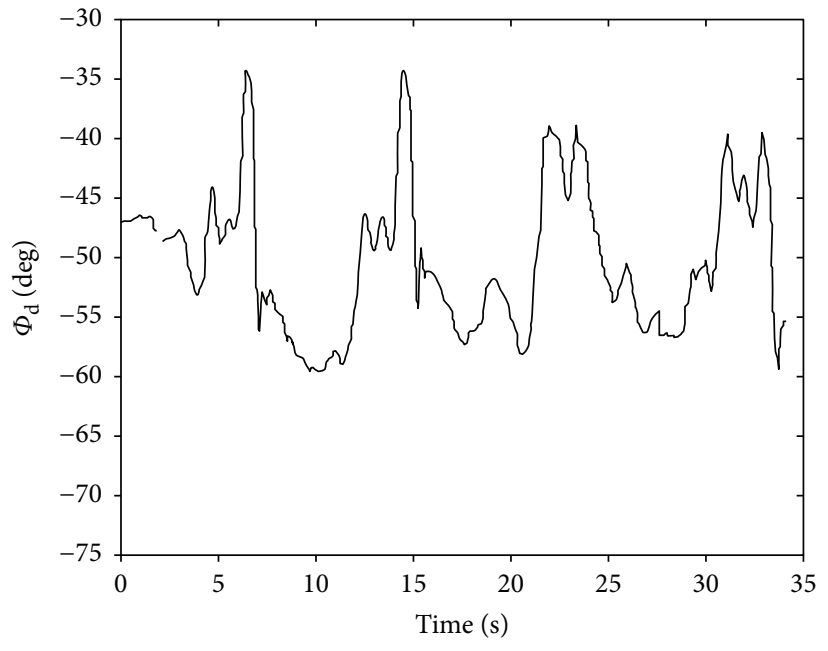

FIgUre 11: The joint angle $\phi_{d}$ that adopted to be the input parameter (ABD).

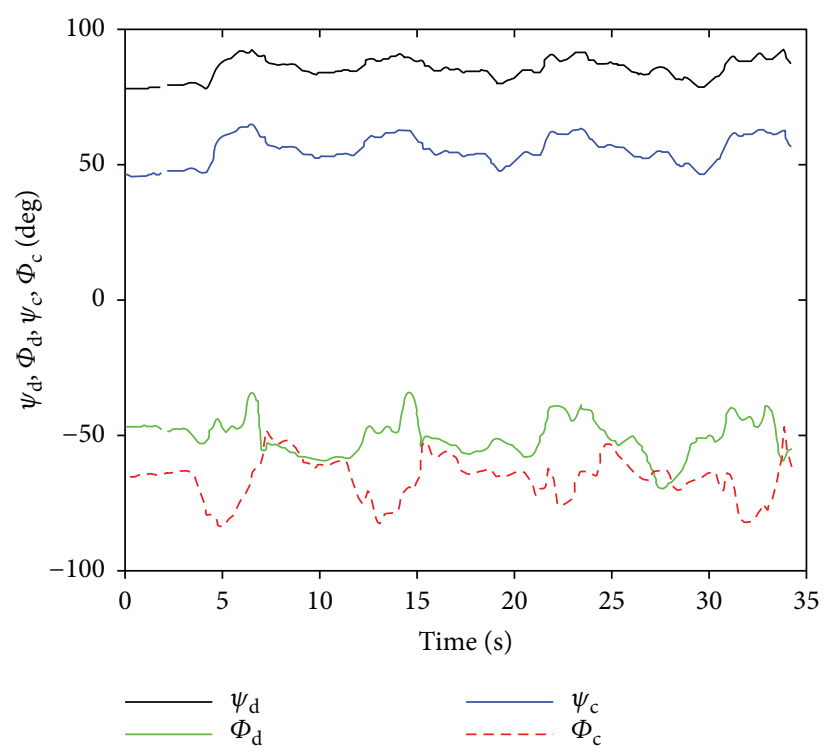

FIGURE 12: The joint angle $\varphi_{d}, \varphi_{c}, \phi_{c}(\mathrm{ABD})$.

For the summary, for the object $S_{1}$ in the ABD motion experiment, the input joint variable $\phi_{d}$ for the position analysis of the mechanism is shown in Figure 11.

Then, entering the joint variables $\theta_{1}, \theta_{2}, \phi_{d}$ into closed solution equations, the joint variables $\varphi_{d}, \varphi_{c}, \phi_{c}$ and $\theta_{4}, \theta_{5}$, $\theta_{6}$ are obtained as shown in Figures 12 and 13.

Thus, the shoulder girdle mechanism position analysis is finished, and the results in the calculated coordinate system of the bone or joint movement posture can be obtained and then converted corresponding to the ISB standard system, so the agency model for shoulder skeletal motion prediction results can be obtained and compared with the experimental measurement results. Figure 14 shows the scapula relative to the thorax motion posture analysis results (ST joint). Figures 15, 16, and 17 are the results of the components of 


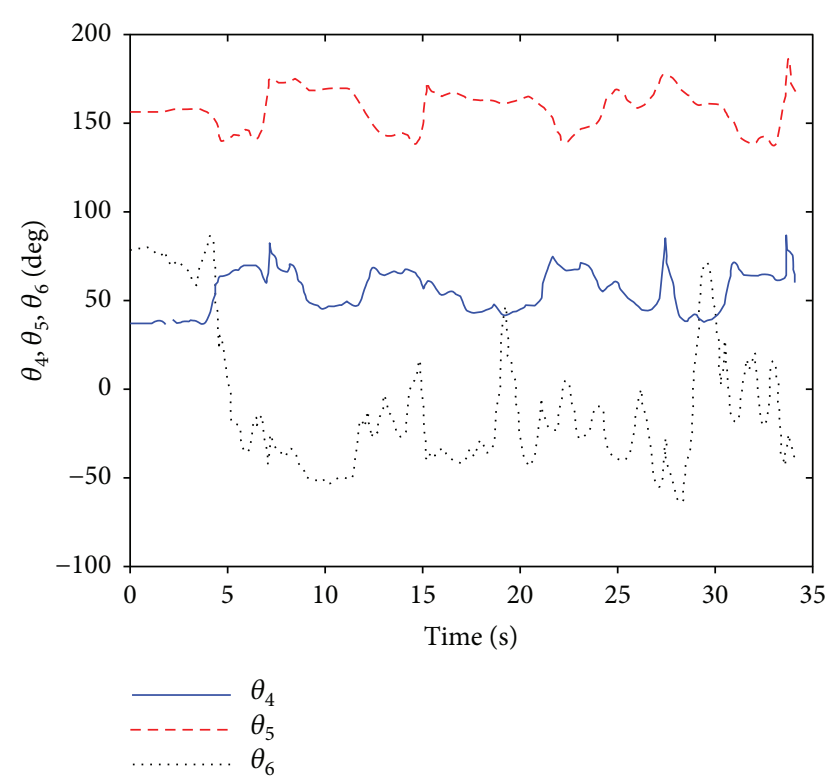

FIgURE 13: The curve of joint angle $\theta_{4}, \theta_{5}, \theta_{6}(\mathrm{ABD})$.

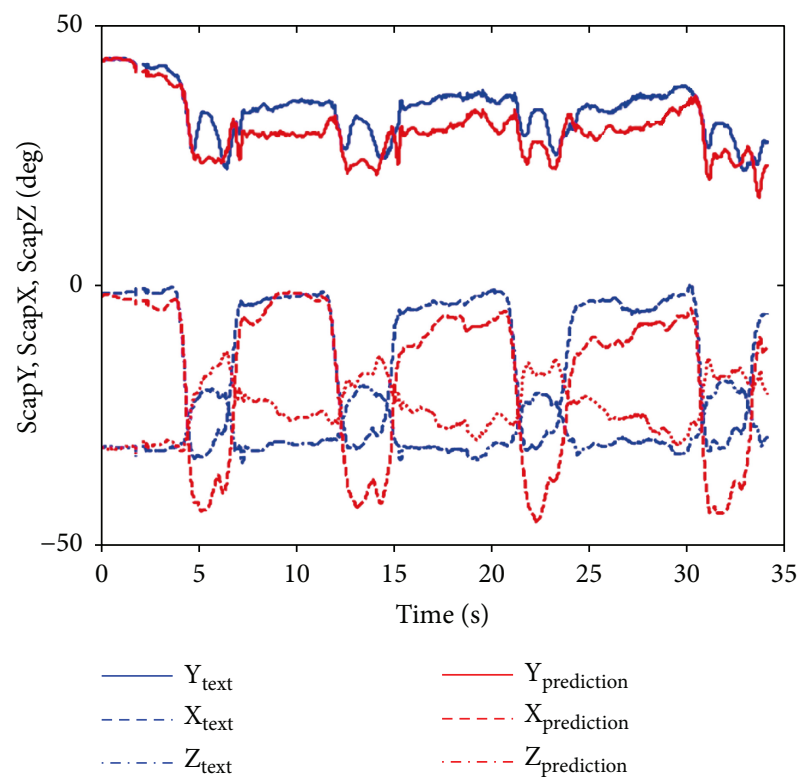

FIgURE 14: The movement of the scapula with respect to the thorax (ABD).

the scapula posture matrix after the decomposition of YXZ rotation sequence according to the ISB standard. Thanks to the fruitful achievement in biological research, the detail and accurate experimental data on shoulder motion can be provided, and in this paper, we selected a set of representative data to verify that the kinematic description of the skeleton model and experimental measurements are consistent.

Using the same method, the exercise experiments of FLEX and SCAP can be analyzed, and the agency model shoulder motion configuration prediction and experimental measurement results are basically consistent.

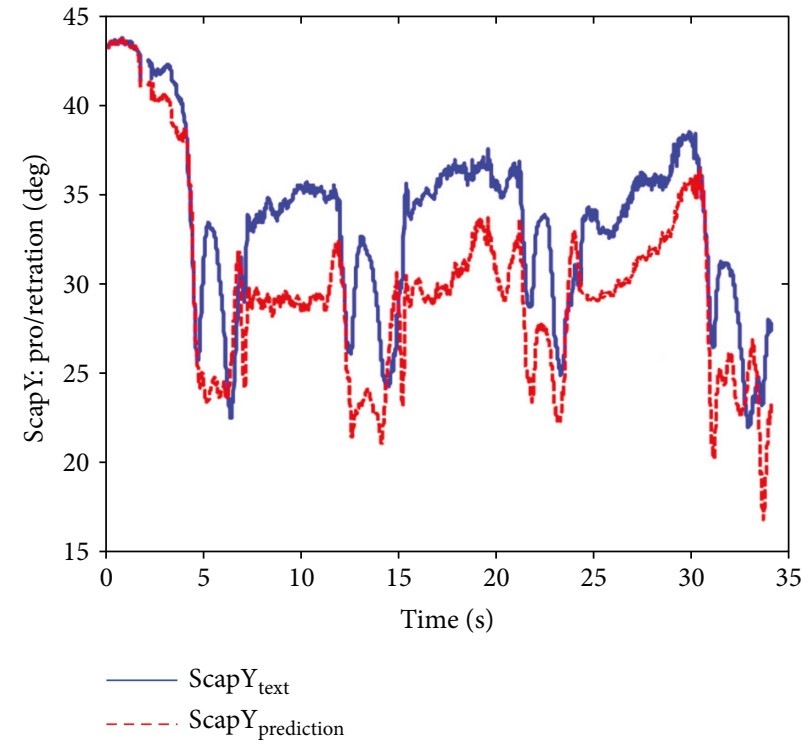

FIGURE 15: Comparison of protraction obtained by prediction and measurement (ABD).

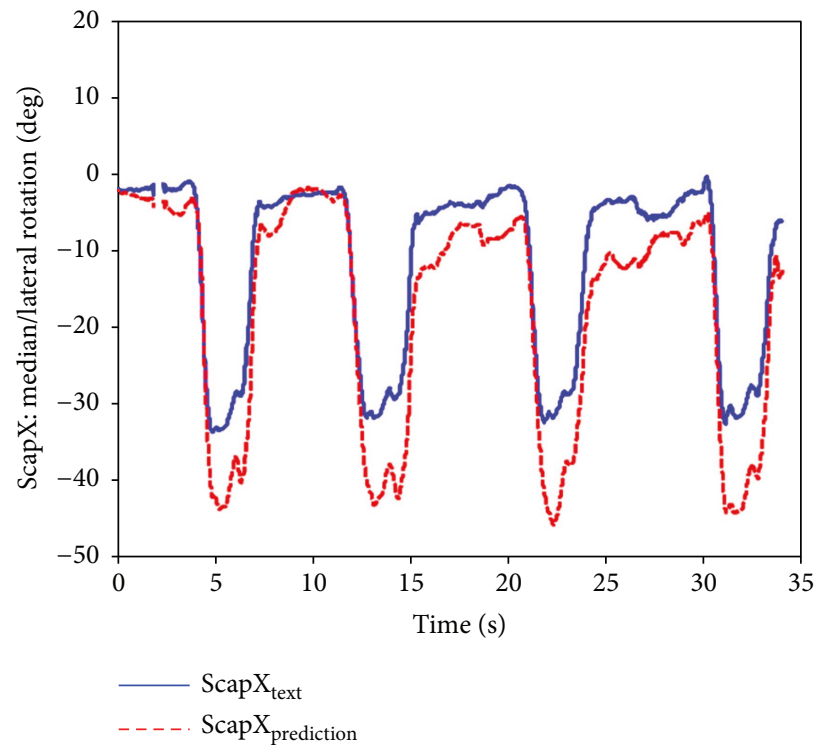

FIGURE 16: Comparison of lateral rotation obtained by prediction and measurement $(\mathrm{ABD})$.

\section{Shoulder Rhythm Model}

From the view of the mechanistic model, the three rotational degrees of freedom of the humerus are independent from the three degrees of freedom of movement of the shoulder girdle mechanism. However, a great number of studies have shown that when the shoulders do some basic movements in the natural state (such as abduction, adduction, external rotation, pronation, flexion, and anteversion), there is a significant correlation and repeatability of shoulder girdle and humeral motion, following the law of the rhythm of the shoulder joint. 


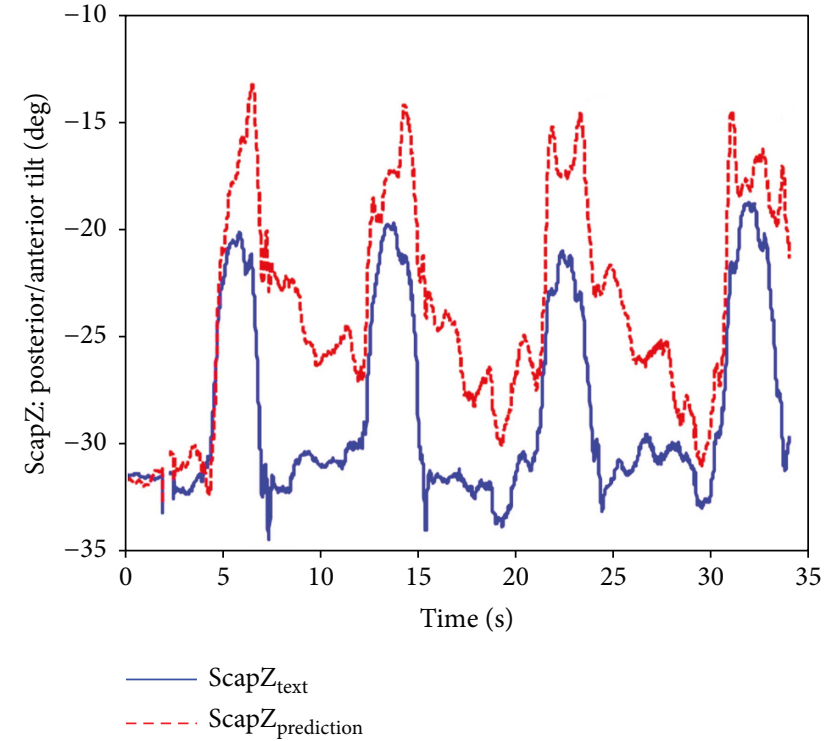

Figure 17: Comparison of anterior tilt obtained by prediction and measurement.

This article simplifies shoulder joint rhythm analysis to the problem of establishing the relationship between the humeral posture and the three independent input joint variables of the shoulder girdle mechanism.

3.1. Rhythm Analysis of Three Kinds of Exercise Task Experiment. The shoulder rhythm of an individual is highly repeatable and less affected by the size of the load when shoulders are in the condition of lighter load [27, 28], but there exist individual differences [29]. Therefore, this paper extends the traditional shoulder joint rhythm and describes the relationship between the scapula and the clavicle posture and the posture of the humerus individually via the proposed model with three independent joint variables $\left(\theta_{1}, \theta_{2}, \phi_{d}\right)$. Through the constraints in the proposed model considering the individual size of shoulder complex, three independent joint variables can be further used to determine the corresponding relationship between the entire shoulder girdle posture and the humeral posture. This method reduces the number of variables involved in rhythms and increases the impact of individual differences.

Studies of shoulder rhythms by the literature [17, 25-28] showed that the elevation plane $\left(\operatorname{HumY}_{1}\right)$ and the elevation (HumX) are relatively independent in shoulder bone movement, while the shoulder girdle posture with two joint variables has a strong regularity. When modeling the shoulder rhythms, this research also uses planes of elevation and elevation as independent variables for rhythm functions.

3.1.1. ABD Sports Rhythm. During the ABD exercise, five actions of lifting and dropping humerus are finished, as shown in Figure 6. Although the subject was asked to do abduction in the frontal plane, the actual elevation of the plane mainly focuses in the scoped of $-10^{\circ}$ to $20^{\circ}$, as shown in Figure 18. Although when the shoulder moved, the joint

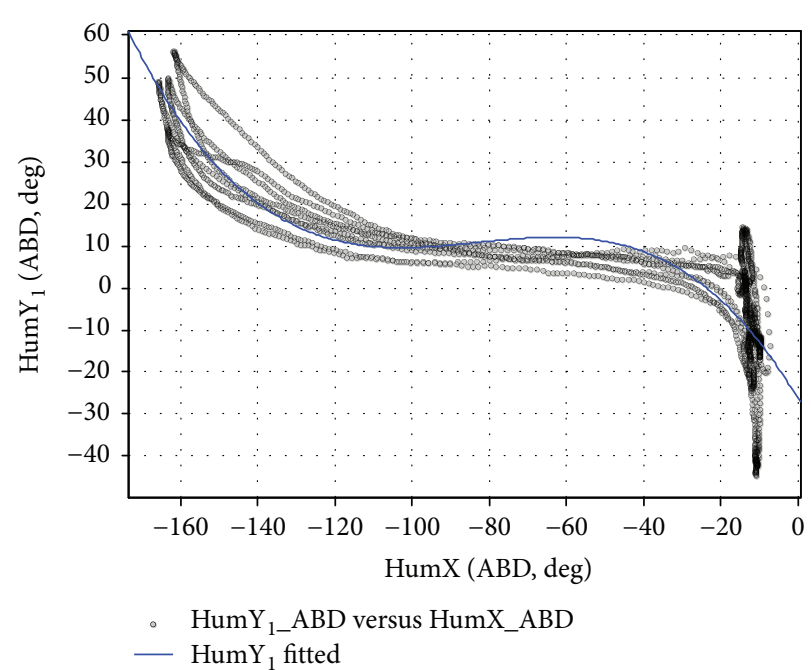

FIgURE 18: The relation between plane of elevation angle and elevation angle (ABD).

variable of elevation plane and the joint variable of elevation are considered independent from each other, the strong regularity and reflexivity are shown in Figure 18, because it represents the physical coordination of the experimental subjects in a certain motion like ABD exercise, and the coupling phenomenon will disappear in arbitrary motion. The relationship between the elevation plane and the elevation in Figure 8 is not universal, so this article will use the plane of elevation and the elevation as two independent variables of the law function.

Figure 19 shows the variation of various dependent variables with the humerus elevation angle when the subject does $\mathrm{ABD}$ exercise. $\theta_{1}, \theta_{2}, \phi_{d}$ is used to determine the rhythm of the shoulder girdle, while Figure 19(d) is used to determine the rhythm of humerus rotation around its shaft $\left(\mathrm{HumY}_{2}\right.$, internal/external rotation). Cubic polynomial fitting is adopted, and shoulder rhythm function can be obtained:

$$
\left[\begin{array}{c}
\theta_{1} \\
\theta_{2} \\
\phi_{d} \\
\text { HumY }_{2}
\end{array}\right]=\left[\begin{array}{c}
{\left[P_{\theta_{1}}\right]_{1 \times 4}} \\
{\left[P_{\theta_{2}}\right]_{1 \times 4}} \\
{\left[P_{\phi_{d}}\right]_{1 \times 4}} \\
{\left[P_{\mathrm{HumY}_{2}}\right]_{1 \times 4}}
\end{array}\right]\left[\begin{array}{c}
(\mathrm{HumX})^{3} \\
(\mathrm{HumX})^{2} \\
(\mathrm{HumX}) \\
1
\end{array}\right],
$$

where $[P]_{1 \times 4}=\left[\begin{array}{llll}p_{1} & p_{2} & p_{3} & p_{4}\end{array}\right]$ denotes the polynomial fitting coefficients to the corresponding joint variables. For simplifying the calculation, the rhythm function corresponding to the $\mathrm{ABD}$ motion is expressed as follows:

$$
f_{\mathrm{ABD}}(\operatorname{HumX})=p_{1}(\operatorname{HumX})^{3}+p_{2}(\operatorname{HumX})^{2}+p_{3}(\operatorname{HumX})+1 .
$$

Table 5 shows the fitting results of the dependent variables, and the accuracy and goodness of the fitting curve 


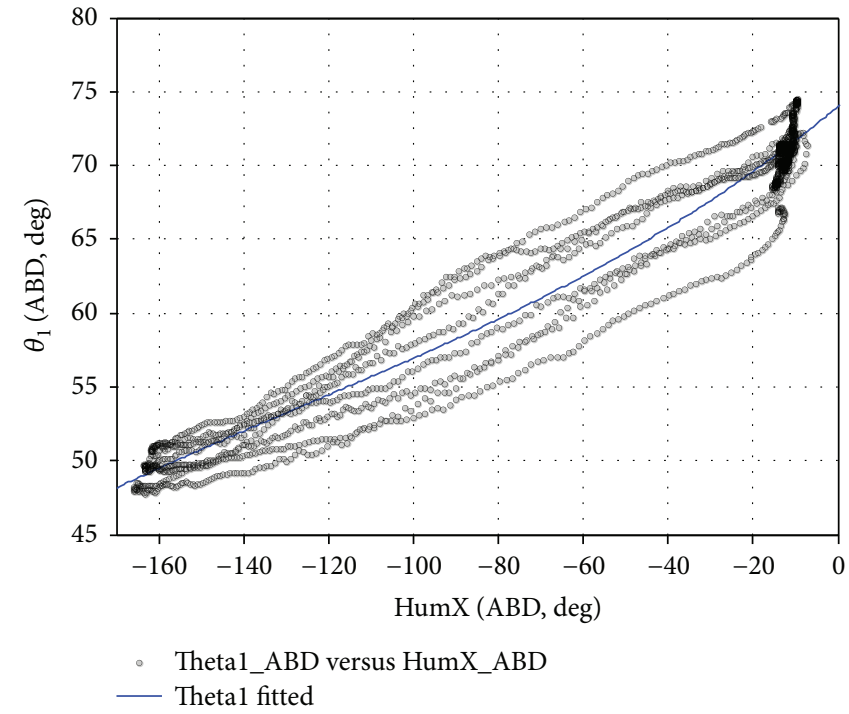

(a) $\theta_{1}$

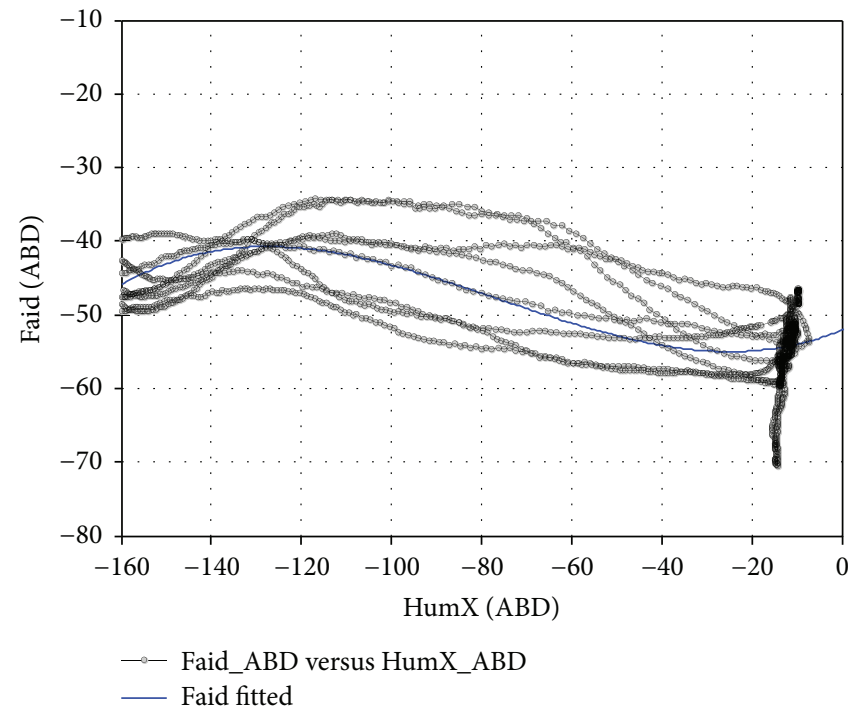

(c) $\phi_{d}$

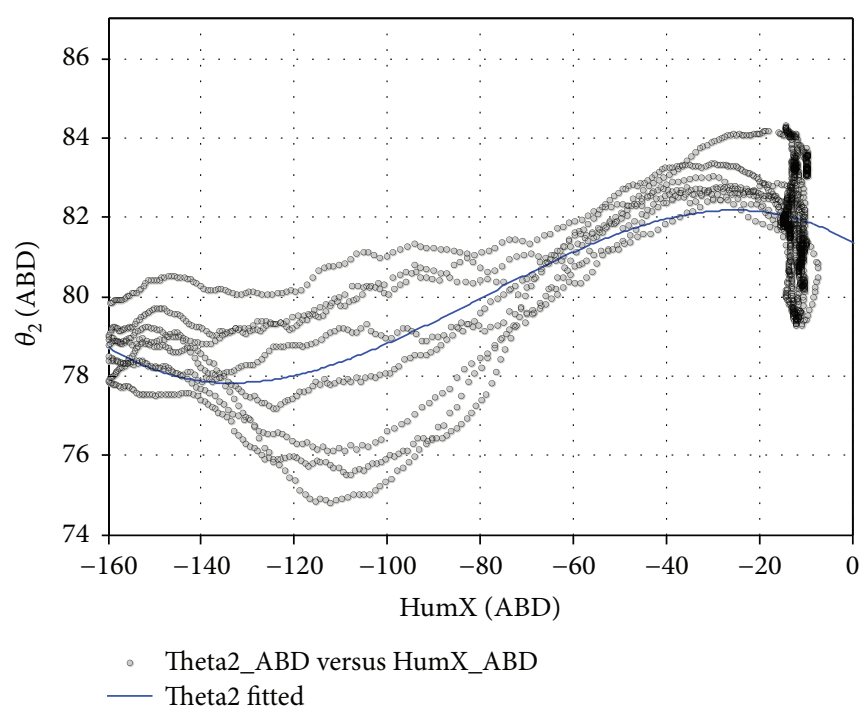

(b) $\theta_{2}$

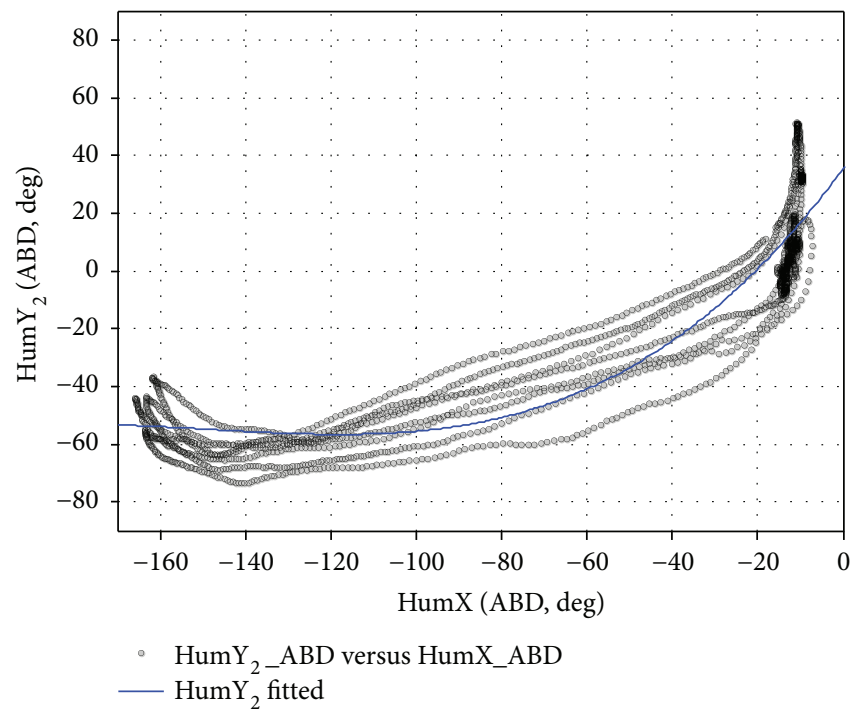

(d) $\mathrm{HumY}_{2}$

FIgURE 19: The joint angles are fit using a third-order polynomial (ABD).

TABLE 5: Third-order polynomial fit parameters of the rhythm function (ABD).

\begin{tabular}{|c|c|c|c|c|c|c|}
\hline \multirow{2}{*}{ Dependent variable joint angle } & \multicolumn{4}{|c|}{ Polynomial coefficients } & \multirow{2}{*}{$\begin{array}{c}R \text {-square } \\
R^{2}\end{array}$} & \multirow{2}{*}{$\begin{array}{c}\text { Deg } \\
\text { RMSE }\end{array}$} \\
\hline & $p_{1}$ & $p_{2}$ & $p_{3}$ & $p_{4}$ & & \\
\hline$\overline{\theta_{1}}$ & $2.483 e-06$ & 0.0009501 & 0.2419 & 74.07 & 0.9581 & 1.811 \\
\hline$\theta_{2}$ & $-6.441 e-06$ & -0.001558 & -0.06742 & 81.4 & 0.6004 & 1.271 \\
\hline$\phi_{d}$ & $2.822 e-05$ & 0.006688 & 0.3163 & -50.89 & 0.5203 & 4.848 \\
\hline $\mathrm{HumY}_{2}$ & $2.443 e-05$ & 0.01201 & 1.833 & 32.07 & 0.8376 & 13.76 \\
\hline
\end{tabular}

are, respectively, measured by root mean square error (RMSE) and coefficient of determination $\left(R^{2}\right)$.

3.1.2. SCAP Sports Rhythm. During the entire SCAP exercise, lifting and dropping actions in the humerus were completed
4 times. To analyze the $\mathrm{ABD}$ motor rhythm, a rhythm function is constructed.

$$
f_{\mathrm{SCAP}}(\operatorname{HumX})=p_{1}(\operatorname{HumX})^{3}+p_{2}(\operatorname{HumX})^{2}+p_{3}(\operatorname{HumX})+1 .
$$




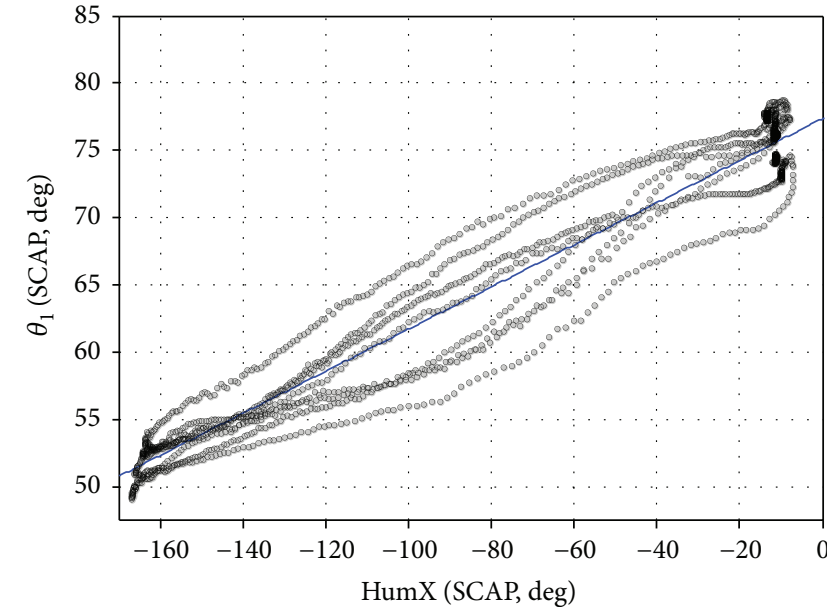

- Theta1_SCAP versus HumX_SCAP - Thetal fitted

(a) $\theta_{1}$

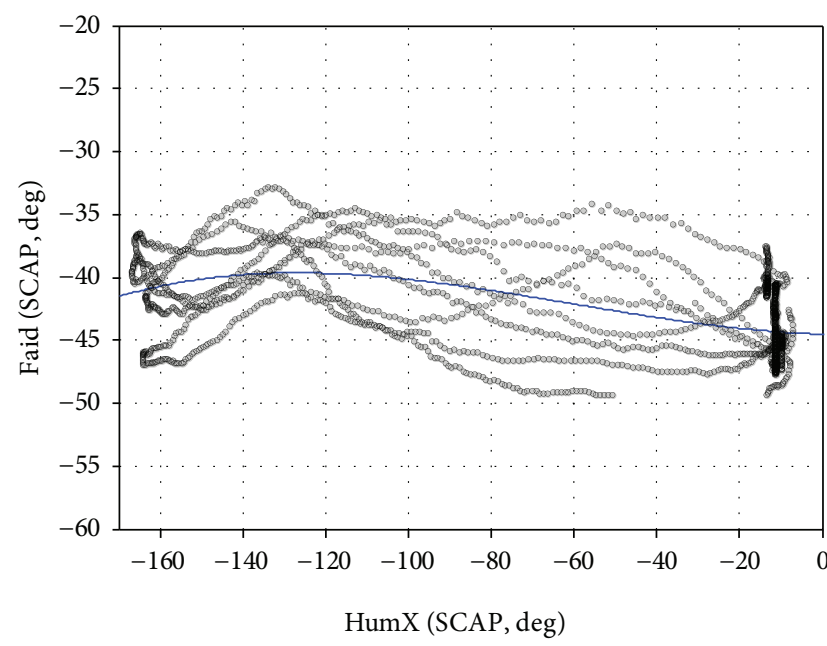

- Faid_SCAP versus HumX_SCAP

— Faid fitted

(c) $\phi_{d}$

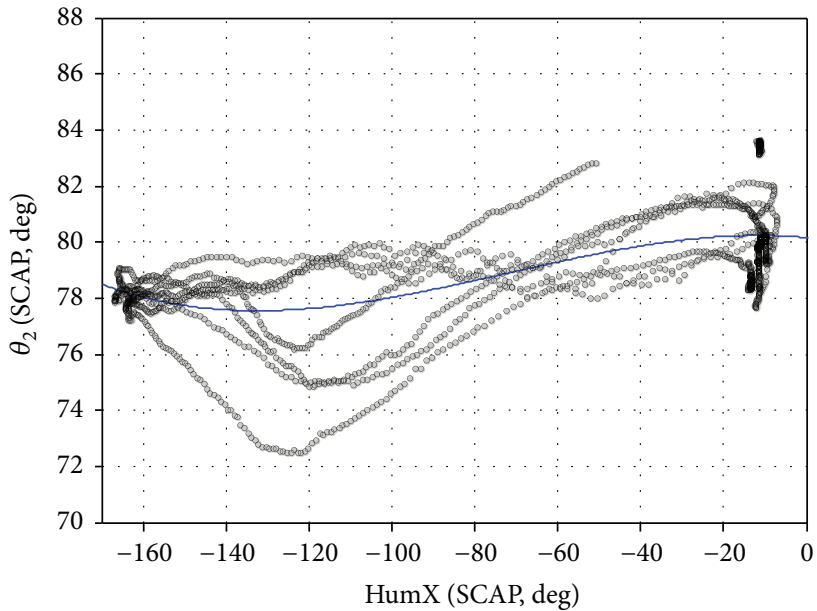

- Theta2_SCAP versus HumX_SCAP

- Theta2 fitted

(b) $\theta_{2}$

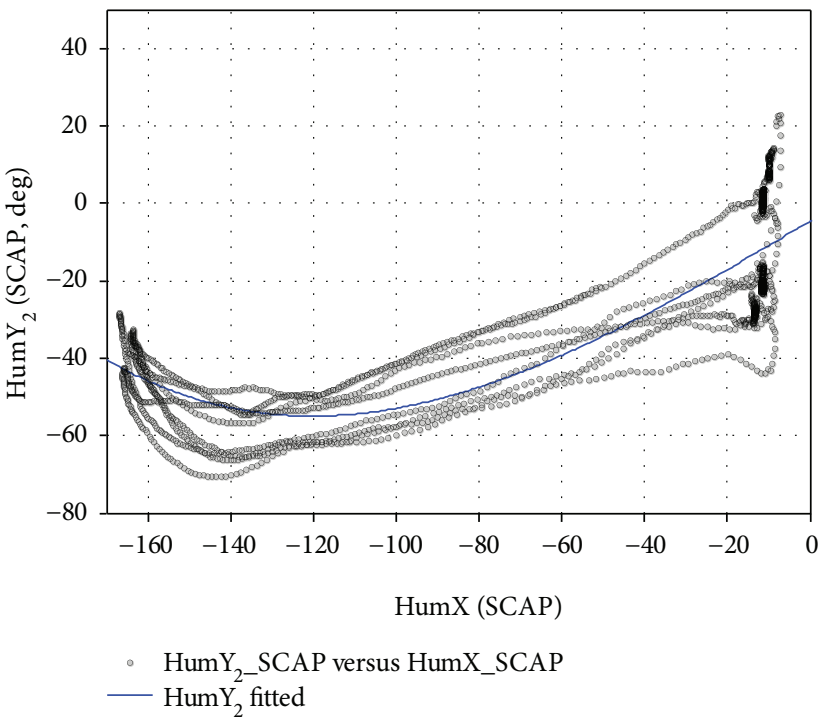

(d) $\mathrm{HumY}_{2}$

FIGURE 20: The joint angles are fit using a third-order polynomial (SCAP).

TABLE 6: Third-order polynomial fit parameters of the rhythm function (SCAP).

\begin{tabular}{|c|c|c|c|c|c|c|}
\hline \multirow{2}{*}{ Dependent variable joint angle } & \multicolumn{4}{|c|}{ Polynomial coefficients } & \multirow{2}{*}{$\begin{array}{c}R \text {-square } \\
R^{2}\end{array}$} & \multirow{2}{*}{$\begin{array}{c}\text { Deg } \\
\text { RMSE }\end{array}$} \\
\hline & $p_{1}$ & $p_{2}$ & $p_{3}$ & $p_{4}$ & & \\
\hline$\theta_{1}$ & $-6.283 e-06$ & -0.001732 & 0.03207 & 76.14 & 0.9574 & 1.922 \\
\hline$\theta_{2}$ & $-2.328 e-07$ & $-3.272 e-06$ & 0.01831 & 80.13 & 0.9161 & 0.6043 \\
\hline$\phi_{d}$ & $4.478 e-06$ & 0.0008388 & -0.002783 & -44.38 & 0.3585 & 2.969 \\
\hline $\mathrm{HumY}_{2}$ & $-1.417 e-05$ & $8.118 e-05$ & 0.6336 & 4.591 & 0.6418 & 12.61 \\
\hline
\end{tabular}

Figure 20 shows the fitting result curve, and Table 6 shows the polynomial rhythm function coefficients, RSME, and coefficients of determination.

3.1.3. FLEX Sports Rhythm. During the entire FLEX exercise, lifting and dropping actions in the humerus were completed
4 times. The same as the ABD motor rhythm analysis, a rhythm function is constructed.

$$
f_{\text {FLEX }}(\operatorname{HumX})=p_{1}(\operatorname{HumX})^{3}+p_{2}(\operatorname{HumX})^{2}+p_{3}(\operatorname{HumX})+1 .
$$




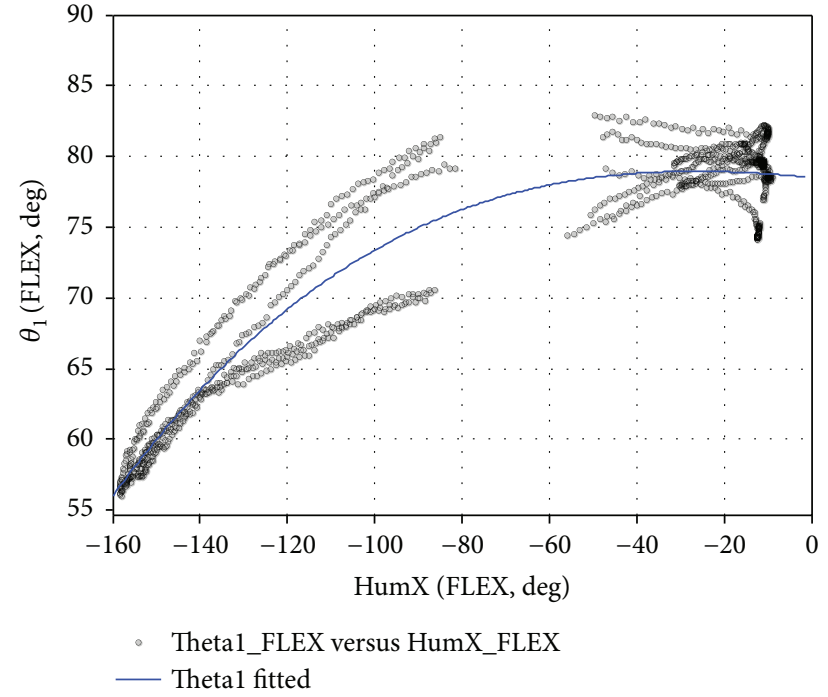

(a) $\theta_{1}$

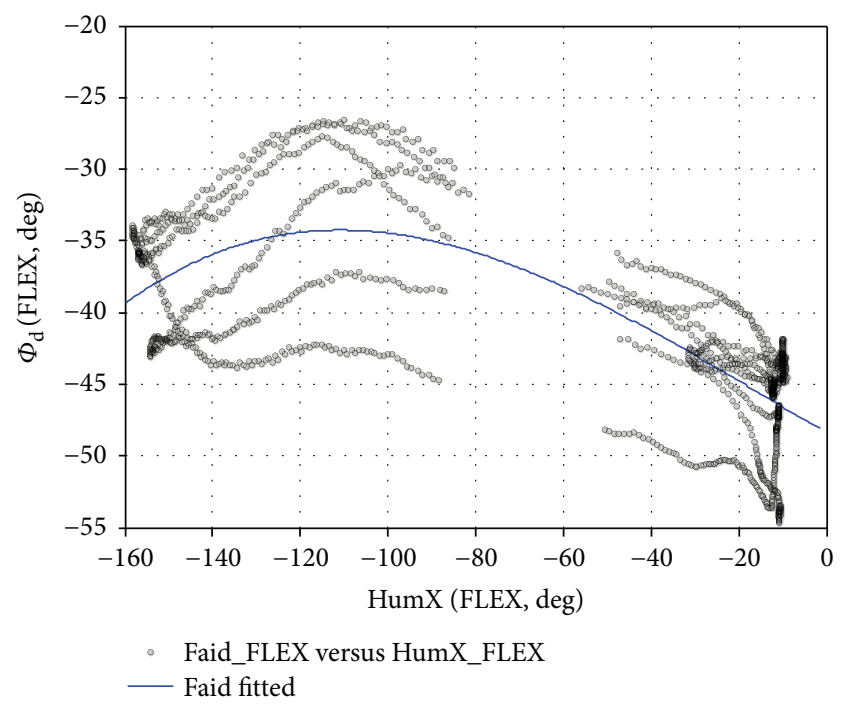

(c) $\phi_{d}$

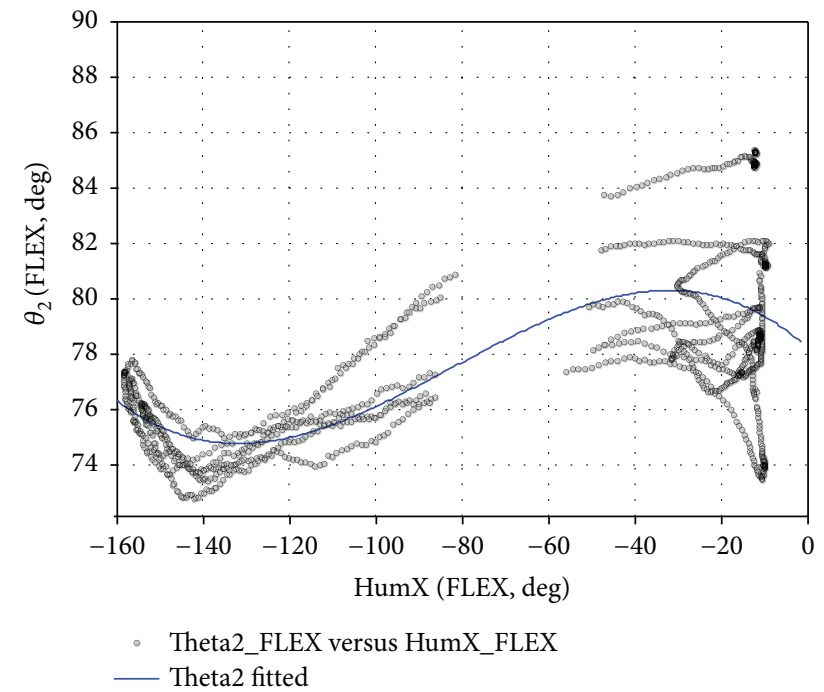

(b) $\theta_{2}$

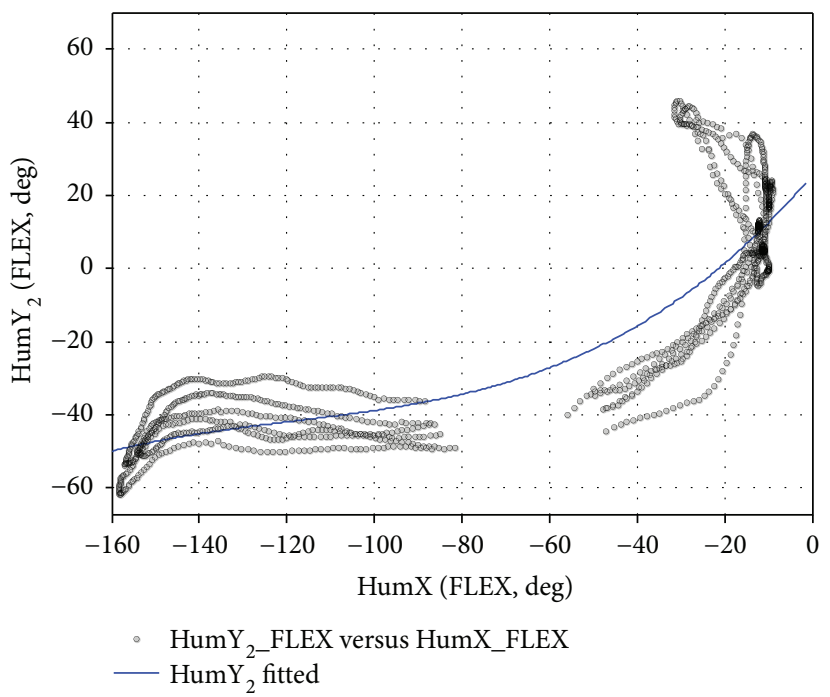

(d) $\mathrm{HumY}_{2}$

FIGURE 21: The joint angles are fit using a third-order polynomial (FLEX).

TABLE 7: Third-order polynomial fit parameters of the rhythm function (FLEX).

\begin{tabular}{|c|c|c|c|c|c|c|}
\hline \multirow{2}{*}{ Dependent variable joint angle } & \multicolumn{4}{|c|}{ Polynomial coefficients } & \multirow{2}{*}{$\begin{array}{c}R \text {-square } \\
R^{2}\end{array}$} & \multirow{2}{*}{$\begin{array}{c}\text { Deg } \\
\text { RMSE }\end{array}$} \\
\hline & $p_{1}$ & $p_{2}$ & $p_{3}$ & $p_{4}$ & & \\
\hline$\theta_{1}$ & $3.587 e-06$ & -0.000406 & -0.01044 & 79.29 & 0.9963 & 0.475 \\
\hline$\theta_{2}$ & $-1.058 e-05$ & -0.002583 & -0.1365 & 77.43 & 0.3921 & 2.833 \\
\hline$\phi_{d}$ & $6.167 e-06$ & 0.0002202 & -0.179 & -48.39 & 0.5784 & 3.951 \\
\hline $\mathrm{HumY}_{2}$ & $3.011 e-05$ & 0.01069 & 1.412 & 25.64 & 0.7932 & 12.75 \\
\hline
\end{tabular}

Figure 21 shows the fitting result curve, and Table 7 shows the polynomial rhythm function coefficient, RSME, and coefficient of determination.

3.2. Shoulder Rhythm Expansion. The lifting plane is not uniquely fixed during ABD, SCAP, and FLEX movements. In the ABD, SCAP, and FLEX, the humerus lifting plane angle Hum $Y_{1}$ changes as shown in Figures 16 and 22. It can be seen that the main movement range of the plane of elevation during these three movements is as shown in Table 8.

Theoretically, the variables of the shoulder joint rhythm function are HumY $Y_{1}$ and HumX in the whole range of activity, and the relationship between the movement of the 


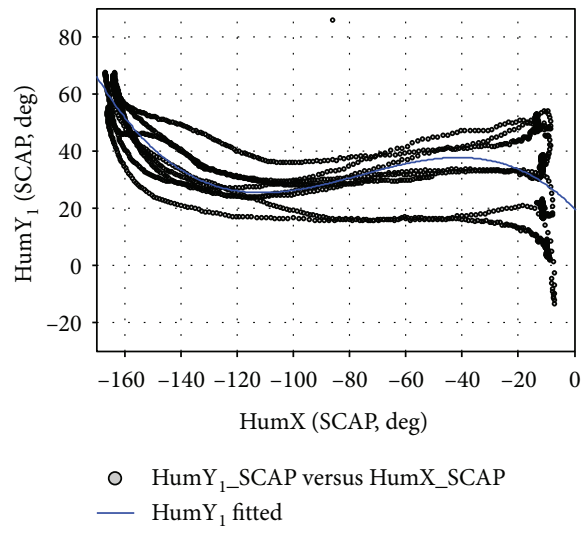

(a)

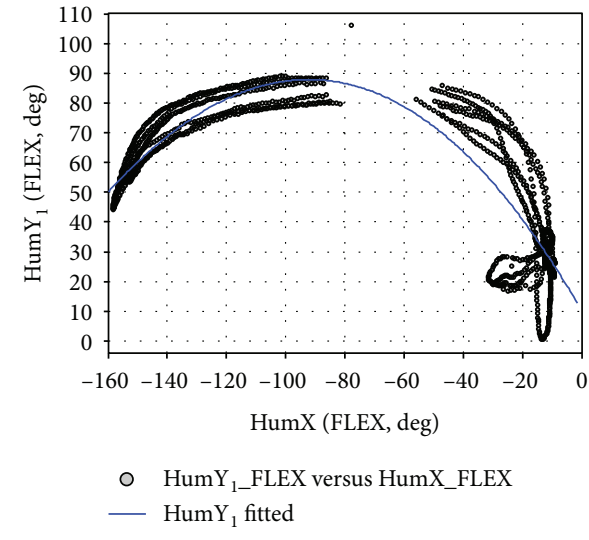

(b)

FIGURE 22: The ranges of plane of elevation when the movement of SCAP and FLEX is exerted.

TABLE 8: The ranges of plane of elevation.

\begin{tabular}{lccc}
\hline Type of exercise & ABD & SCAP & FLEX \\
\hline Range of plane of elevation & $-10^{\circ} \sim 20^{\circ}$ & $20^{\circ} \sim 50^{\circ}$ & $50^{\circ} \sim 90^{\circ}$ \\
\hline
\end{tabular}

shoulder bones and the axial rotation angle $\mathrm{HumY}_{2}$ of the humerus is not significant. At the same time, axial rotation angles HumY $\mathrm{Y}_{2}$ and HumX are related. Therefore, in this research, the shoulder joint rhythms corresponding to $\mathrm{ABD}, \mathrm{SCAP}$, and FLEX are extended to the motion space $-10^{\circ} \leq \operatorname{HumY}_{1} \leq 90^{\circ}$ and $0^{\circ} \leq \operatorname{HumX} \leq 165^{\circ}$, and the rhythm can be expressed as follows:

$$
f(\operatorname{HumX})= \begin{cases}f_{\mathrm{ABD}}(\operatorname{HumX}), & -10^{\circ} \leq \mathrm{HumY}_{1} \leq 20^{\circ}, \\ f_{\mathrm{SCAP}}(\operatorname{HumX}), & 20^{\circ} \leq \mathrm{HumY}_{1} \leq 50^{\circ}, 0^{\circ} \leq \operatorname{HumX} \leq 165^{\circ}, \\ f_{\mathrm{FLEX}}(\operatorname{HumX}), & 50^{\circ} \leq \mathrm{HumY}_{1} \leq 90^{\circ} .\end{cases}
$$

\section{Conclusion}

In this paper, a new shoulder complex skeletal model based on joint geometric constraints is proposed using a spatial hybrid mechanism to describe the movement of human shoulder complex, which is developed for the rehabilitation robots. The effectiveness and viability of the proposed model have been verified via the experimental data of shoulder skeletal motion. Based on this model, through the analysis of the angle and change rule of each joint in the shoulder complex during the movement, the shoulder rhythm was obtained by means of polynomial fitting with $\mathrm{HumY}_{1}$ and HumX as independent variables to make it suitable to guide the training strategy for shoulder rehabilitation via robotics.

\section{Conflicts of Interest}

The authors declare that they have no conflicts of interest.

\section{Acknowledgments}

This work was supported by the Natural Science Foundation of China (Project nos. 51475322, 51535008, 51775367, and 51721003), Tianjin Municipal Science and Technology Department Program (Grant no. 17JCZDJC30300), and the Programme of Introducing Talents of Discipline to Universities ("111 Program”) under Grant no. B16034.

\section{References}

[1] D. K. Zondervan, L. Palafox, J. Hernandez, and D. J. Reinkensmeyer, "The resonating arm xerciser: design and pilot testing of a mechanically passive rehabilitation device that mimics robotic active assistance," Journal of Neuroengineering and Rehabilitation, vol. 10, p. 39, 2013.

[2] S. Barreca, S. L. Wolf, S. Fasoli, and R. Bohannon, "Treatment interventions for the paretic upper limb of stroke survivors: a critical review," Neurorehabilitation and Neural Repair, vol. 17, no. 4, pp. 220-226, 2003.

[3] H. M. Feys, W. J. de Weerdt, B. E. Selz et al., "Effect of a therapeutic intervention for the hemiplegic upper limb in the acute phase after stroke : a single-blind, randomized, controlled multicenter trial," Stroke, vol. 29, no. 4, pp. 785-792, 1998.

[4] R. Colombo, F. Pisano, S. Micera et al., "Robotic techniques for upper limb evaluation and rehabilitation of stroke patients," IEEE Transactions on Neural Systems and Rehabilitation Engineering, vol. 13, no. 3, pp. 311-324, 2005. 
[5] P. S. Lum, C. G. Burgar, P. C. Shor, M. Majmundar, and M. van der Loos, "Robot-assisted movement training compared with conventional therapy techniques for the rehabilitation of upper-limb motor function after stroke," Archives of Physical Medicine and Rehabilitation, vol. 83, no. 7, pp. 952-959, 2002.

[6] D. J. Reinkensmeyer, J. L. Emken, and S. C. Cramer, "Robotics, motor learning, and neurologic recovery," Annual Review of Biomedical Engineering, vol. 6, no. 1, pp. 497-525, 2004.

[7] R. Riener, T. Nef, and G. Colombo, "Robot-aided neurorehabilitation of the upper extremities," Medical and Biological Engineering and Computing, vol. 43, no. 1, pp. 2-10, 2005.

[8] C. Carignan and M. Liszka, "Design of an arm exoskeleton with scapula motion for shoulder rehabilitation," in 2005. ICAR '05. Proceedings., 12th International Conference on Advanced Robotics, pp. 524-531, Seattle, WA, USA, July 2005.

[9] W. T. Dempster, "Mechanisms of shoulder movement," Archives of Physical Medicine and Rehabilitation, vol. 46, pp. 49-70, 1965.

[10] A. E. Engin and S. M. Chen, "Statistical data base for the biomechanical properties of the human shoulder complex-I: kinematics of the shoulder complex," Journal of Biomechanical Engineering, vol. 108, no. 3, pp. 215-221, 1986.

[11] B. A. Garner and M. G. Pandy, "Musculoskeletal model of the upper limb based on the visible human male dataset," Computer Methods in Biomechanics and Biomedical Engineering, vol. 4, no. 2, pp. 93-126, 2001.

[12] W. Maurel and D. Thalmann, "Human shoulder modeling including scapulo-thoracic constraint and joint sinus cones," Computers \& Graphics, vol. 24, no. 2, pp. 203-218, 2000.

[13] W. Maurel, D. Thalmann, P. Hoffmeyer et al., "A biomechanical musculoskeletal model of human upper limb for dynamic simulation," in Computer Animation and Simulation '96. Eurographics, R. Boulic and G. Hégron, Eds., pp. 121-136, Springer, Vienna, 1996.

[14] B. Tondu, "Modelling of the shoulder complex and application the design of upper extremities for humanoid robots," in 2005 5th IEEE-RAS International Conference on Humanoid Robots, pp. 313-320, Tsukuba, Japan, December 2005.

[15] J. Lenarcic and M. Stanisic, "A humanoid shoulder complex and the humeral pointing kinematics," IEEE Transactions on Robotics \& Automation, vol. 19, no. 3, pp. 499-506, 2003.

[16] J. Klein, S. Spencer, J. Allington, J. E. Bobrow, and D. J. Reinkensmeyer, "Optimization of a parallel shoulder mechanism to achieve a high-force, low-mass, robotic-arm exoskeleton," IEEE Transactions on Robotics, vol. 26, no. 4, pp. 710-715, 2010.

[17] B. A. Garner and M. G. Pandy, "A kinematic model of the upper limb based on the visible human project (VHP) image dataset," Computer Methods in Biomechanics and Biomedical Engineering, vol. 2, no. 2, pp. 107-124, 1999.

[18] H. Zhen, Z. Yongsheng, and Z. Tieshi, Institution of Higher Space, Higher Education Press, Beijing, 2014.

[19] C. G. M. Meskers, H. M. Vermeulen, J. H. de Groot, F. C. T. van der Helm, and P. M. Rozing, "3D shoulder position measurements using a six-degree-of-freedom electromagnetic tracking device," Clinical Biomechanics, vol. 13, no. 4-5, pp. 280-292, 1998.

[20] F. C. T. van der Helm and G. M. Pronk, "Three-dimensional recording and description of motions of the shoulder mechanism," Journal of Biomechanical Engineering, vol. 117, no. 1, pp. 27-40, 1995.
[21] B. Bolsterlee, H. E. J. Veeger, and F. C. T. van der Helm, "Modelling clavicular and scapular kinematics: from measurement to simulation," Medical \& Biological Engineering \& Computing, vol. 52, no. 3, pp. 283-291, 2014.

[22] G. Wu and P. R. Cavanagh, "ISB recommendations for standardization in the reporting of kinematic data," Journal of Biomechanics, vol. 28, no. 10, pp. 1257-1261, 1995.

[23] G. Wu, F. van der Helm, H. E. Veeger et al., "ISB recommendation on definitions of joint coordinate systems of various joints for the reporting of human joint motion-part II: shoulder, elbow, wrist and hand," Journal of Biomechanics, vol. 38, no. 5, pp. 981-992, 2005.

[24] B. A. Garner and M. G. Pandy, "The obstacle-set method for representing muscle paths in musculoskeletal models," Computer Methods in Biomechanics and Biomedical Engineering, vol. 3, no. 1, pp. 1-30, 2000.

[25] M. D. Klein Breteler, C. W. Spoor, and F. C. T. van der Helm, "Measuring muscle and joint geometry parameters of a shoulder for modeling purposes," Journal of Biomechanics, vol. 32, no. 11, pp. 1191-1197, 1999.

[26] A. A. Nikooyan, H. E. J. Veeger, P. Westerhoff et al., “An EMG-driven musculoskeletal model of the shoulder," Human Movement Science, vol. 31, no. 2, pp. 429-447, 2012.

[27] C. Högfors, B. Peterson, G. Sigholm, and P. Herberts, "Biomechanical model of the human shoulder joint-II. The shoulder rhythm," Journal of Biomechanics, vol. 24, no. 8, pp. 699709, 1991.

[28] P. W. McClure, L. A. Michener, B. J. Sennett, and A. R. Karduna, "Direct 3-dimensional measurement of scapular kinematics during dynamic movements in vivo," Journal of Shoulder and Elbow Surgery, vol. 10, no. 3, pp. 269-277, 2001.

[29] M. B. Warner, P. H. Chappell, and M. J. Stokes, "Measurement of dynamic scapular kinematics using an acromion marker cluster to minimize skin movement artifact," Journal of Visualized Experiments Jove, vol. 96, no. 96, article e51717, 2015. 


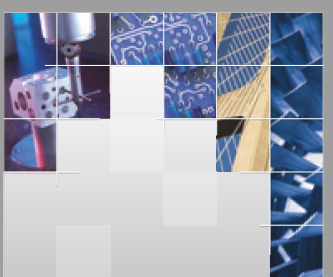

\section{Enfincering}
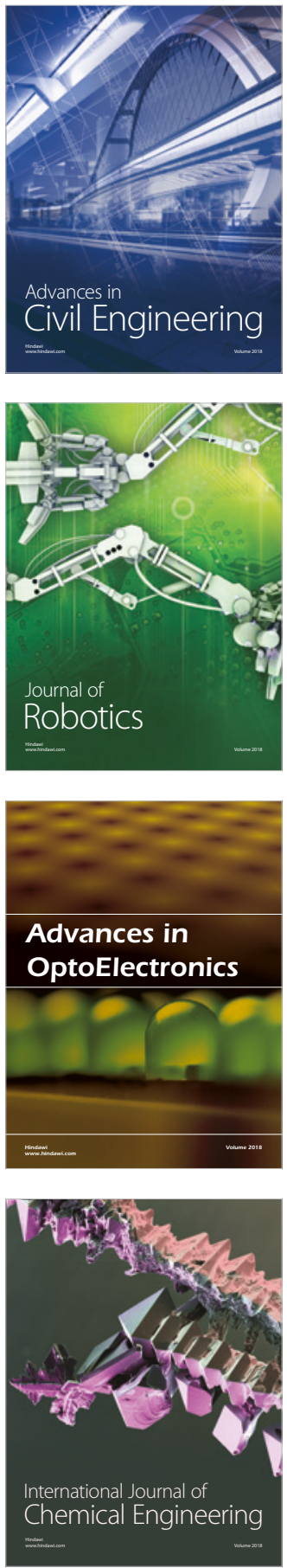

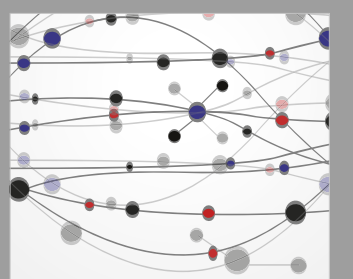

\section{Rotating \\ Machinery}

The Scientific World Journal

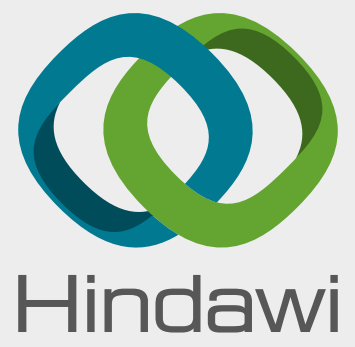

Submit your manuscripts at

www.hindawi.com
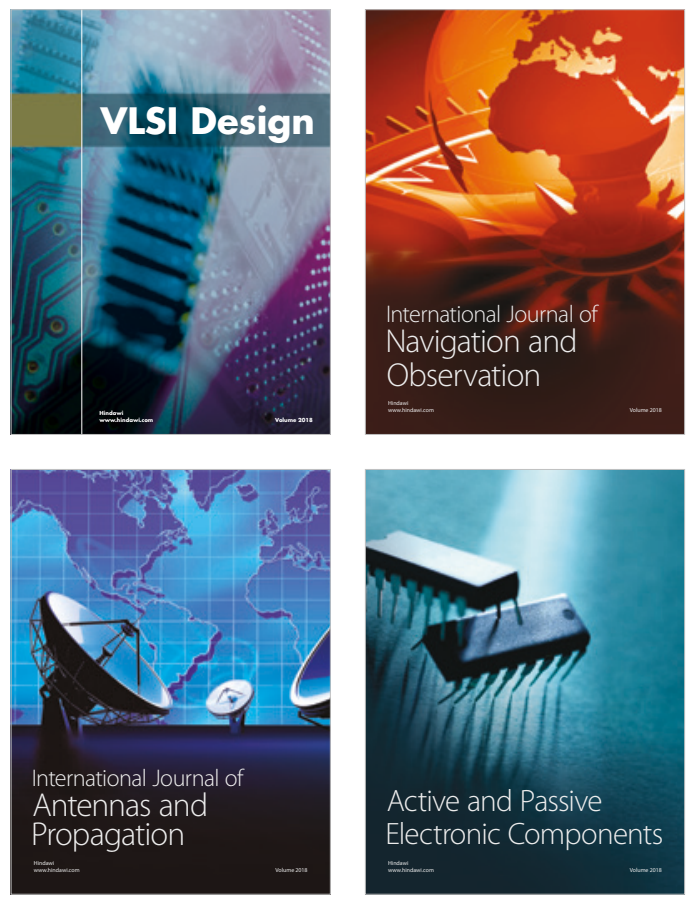
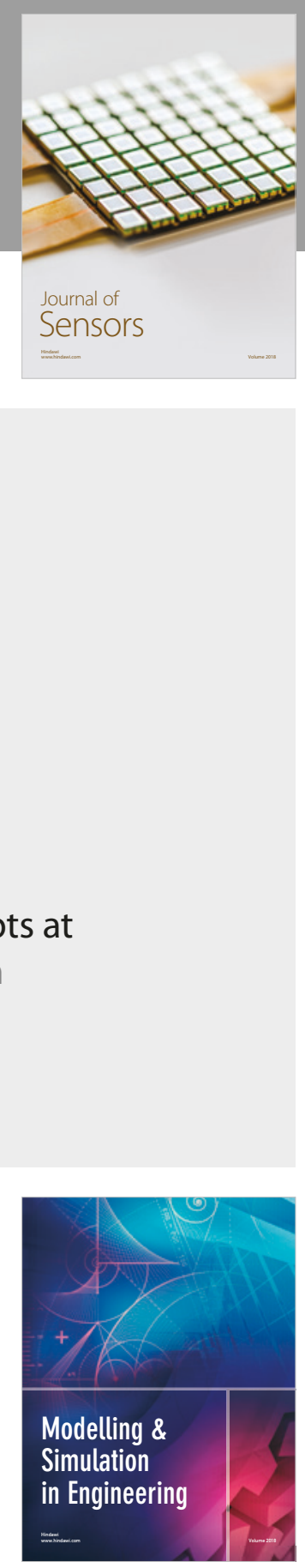

\section{Advances \\ Multimedia}
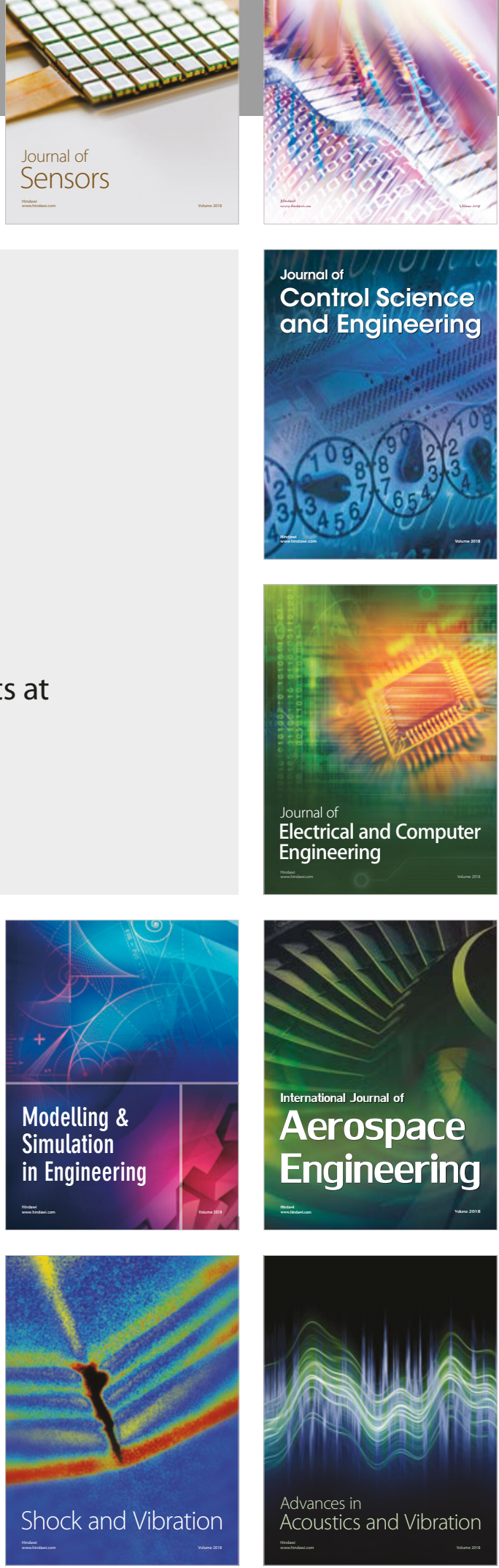